\title{
Multiple scales and singular limits for compressible rotating fluids with general initial data
}

\author{
Eduard Feireisl* Antonín Novotný \\ Institute of Mathematics of the Academy of Sciences of the Czech Republic \\ Žitná 25, 11567 Praha 1, Czech Republic \\ Charles University in Prague, Faculty of Mathematics and Physics, Mathematical Institute \\ Sokolovská 83, 18675 Praha 8, Czech Republic \\ and \\ IMATH, EA 2134, Université du Sud Toulon-Var BP 20132, 83957 La Garde, France
}

\begin{abstract}
We study the singular limit of a rotating compressible fluid described by a scaled barotropic Navier-Stokes system, where the Rossby number $=\varepsilon$, the Mach number $=\varepsilon^{m}$, the Reynolds number $=\varepsilon^{-\alpha}$, and the Froude number $=\varepsilon^{n}$ are proportional to a small parameter $\varepsilon \rightarrow 0$. The inviscid planar Euler system is identified as the limit problem. The proof is based on the application of the method of relative entropies and careful analysis of oscillatory integrals describing the propagation of Rossby-acoustic waves.
\end{abstract}

\section{Introduction}

The basic system of equations considered in this paper are the continuity equation and the momentum equation describing the time evolution of the mass density $\varrho=\varrho(t, x)$ and the (relative) velocity $\mathbf{u}=\mathbf{u}(t, x)$ of a compressible, rotating fluid:

${ }^{*}$ Eduard Feireisl acknowledges the support of the project LL1202 in the programme ERC-CZ funded by the Ministry of Education, Youth and Sports of the Czech Republic. 


$$
\begin{gathered}
\partial_{t} \varrho+\operatorname{div}_{x}(\varrho \mathbf{u})=0, \\
\partial_{t}(\varrho \mathbf{u})+\operatorname{div}_{x}(\varrho \mathbf{u} \otimes \mathbf{u})+\frac{1}{\varepsilon} \varrho(\mathbf{f} \times \mathbf{u})+\frac{1}{\varepsilon^{2 m}} \nabla_{x} p(\varrho)=\varepsilon^{\alpha} \operatorname{div}_{x} \mathbb{S}\left(\nabla_{x} \mathbf{u}\right)+\frac{1}{\varepsilon^{2 n}} \varrho \nabla_{x} G, \\
\mathbb{S}\left(\nabla_{x} \mathbf{u}\right)=\mu\left(\nabla_{x} \mathbf{u}+\nabla_{x}^{t} \mathbf{u}-\frac{2}{3} \operatorname{div}_{x} \mathbf{u I}\right)+\eta \operatorname{div}_{x} \mathbf{u I}, \mu>0, \eta \geq 0 .
\end{gathered}
$$

The fluid is confined to an infinite slab

$$
\Omega=R^{2} \times(0,1)
$$

where it satisfies the slip condition

$$
\left.\mathbf{u} \cdot \mathbf{n}\right|_{\partial \Omega}=\left.\left[\mathbb{S}\left(\nabla_{x} \mathbf{u}\right) \cdot \mathbf{n}\right]_{\tan }\right|_{\partial \Omega}=0
$$

imposed on the horizontal boundary.

The model (1.1 - 1.5) may be viewed as a crude approximation ( $f$-plane model) of the Earth atmosphere in a plane tangent to the Earth at a certain latitude, see Vallis [34, Chapter 2, Section 2.3]. Accordingly, the gravitational force is taken parallel to the vertical projection of the rotation axis:

$$
\mathbf{f}=[0,0,1], \nabla_{x} G=[0,0,-1],
$$

The momentum equation (1.2) contains a small parameter $\varepsilon$ related to different characteristic numbers resulting from the scale analysis: Rossby number $=\varepsilon$, Mach number $=\varepsilon^{m}$, Reynolds number $=\varepsilon^{-\alpha}$, Froude number $=\varepsilon^{n}$, see Klein [20]. We consider the singular limit problem for $\varepsilon \searrow 0$ in the multiscale regime:

$$
\frac{m}{2}>n \geq 1, \alpha>0
$$

for the ill-prepared initial data

$$
\varrho(0, \cdot)=\varrho_{0, \varepsilon}=\tilde{\varrho}_{\varepsilon}+\varepsilon^{m} \varrho_{\varepsilon}^{(1)}, \mathbf{u}(0, \cdot)=\mathbf{u}_{0}
$$

where $\tilde{\varrho}_{\varepsilon}$ is a solution to the static problem

$$
\nabla_{x} p\left(\tilde{\varrho}_{\varepsilon}\right)=\varepsilon^{2(m-n)} \tilde{\varrho}_{\varepsilon} \nabla_{x} G \text { in } \Omega .
$$

In particular, since $n \geq 1$, the centrifugal force, parallel to the vertical axis, is dominated by gravitation (cf. Durran [7]). 
Formally, it is not difficult to identify the limit problem. Indeed fast rotation is expected to eliminate the vertical motion, the vanishing viscosity (high Reynolds number) makes the limit system inviscid (hyperbolic), while the low Mach number regime drives the fluid to incompressibility. The limit problem is therefore expected to be the incompressible Euler system for the planar velocity field $\mathbf{v}=\left[v_{1}, v_{2}\right]$,

$$
\partial_{t} \mathbf{v}+\operatorname{div}_{x}(\mathbf{v} \otimes \mathbf{v})+\nabla_{x} \Pi=0, \operatorname{div}_{x} \mathbf{v}=0 \text { in }(0, T) \times R^{2} .
$$

Our main goal is to put these formal arguments on rigorous grounds.

The phenomena discussed above have been investigated by many authors. The fact that highly rotating fluids become planar (two-dimensional), and, accordingly, fast rotation has a regularizing effect, was observed by Babin, Mahalov, and Nicolaenko [1], [2], Bresch, Desjardins, and GerardVaret, [3], Chemin et al. [4], among others. The inviscid limit is a well studied and partially still open challenging problem, see Clopeau, Mikelic, Robert [5], Kato [17], Masmoudi [23], [24], 25], Sammartino and Caflisch [27], [28], Swann [31], Temam and Wang [32], [33], to name only a few. The low Mach number limits were proposed in the pioneering papers by Ebin [8], and Klainerman and Majda [19], and later reexamined in the context of weak solutions by Lions and Masmoudi [22], see also the survey by Danchin [6], Gallagher [14], and Schochet [29]. To the best of our knowledge, the simultaneous effects of these three mechanisms has not yet been treated in the literature.

The present paper may be viewed as complementary to our previous study [11, where we examined the "single-scale" limit corresponding to

$$
n=0, m=1, \alpha>0 \text {. }
$$

Although this problem looks formally very similar to the present setting, the methods employed as well as the limit system are different, cf. [11]. The central issue to be discussed is the behavior of the oscillatory part of solutions to the scaled system. These are described in the case (1.10) by a system of linear equations with constant coefficients, while the situation (1.6) gives rise to a problem with coefficients depending on the scaling parameter $\varepsilon$.

Similarly to [11], our approach is based on the concept of finite energy weak solutions satisfying the relative entropy inequality identified in [13], see Section 2. After collecting the necessary preliminary material, we state the main result in Section 3. Section 4 reviews the basic estimates, independent of $\varepsilon \searrow 0$, for solutions of the family of scaled problems. Section 5 is the heart of the paper. We establish decay estimates on the oscillatory part of solutions to the scaled problems by means of careful analysis of certain oscillatory integrals. Here, inspired by the analysis of Guo, Peng, and Wang [16], we make use of frequency cut-off operators and estimates based on van Corput's lemma. The proof of convergence towards the limit system is completed in Section 6 , 


\section{Preliminaries, weak solutions, relative entropy inequality}

We suppose that the pressure $p \in C[0, \infty) \cap C^{3}(0, \infty)$ is a given function of the density enjoying the following property

$$
p(0)=0, p^{\prime}(\varrho)>0 \text { for all } \varrho>0, \lim _{\varrho \rightarrow \infty} \frac{p^{\prime}(\varrho)}{\varrho^{\gamma-1}}, \gamma>\frac{3}{2} .
$$

In addition, without loss of generality, we assume that $p$ is "normalized" setting

$$
p^{\prime}(1)=1 \text {. }
$$

Finally, we introduce the pressure potential $H$,

$$
H(\varrho)=\varrho \int_{1}^{\varrho} \frac{p(z)}{z^{2}} \mathrm{~d} z
$$

noting that

$$
H^{\prime \prime}(\varrho)=\frac{p^{\prime}(\varrho)}{\varrho}, H^{\prime \prime}(1)=1
$$

\subsection{Static solutions}

As already mentioned above, the static solutions $\tilde{\varrho}_{\varepsilon}$ solve the problem (1.8), specifically, we take

$$
H^{\prime}\left(\tilde{\varrho}_{\varepsilon}\right)=\varepsilon^{2(m-n)} G+H^{\prime}(1), \text { where } G(x)=-x_{3} ;
$$

whence

$$
\tilde{\varrho}_{\varepsilon}(x)=\tilde{\varrho}_{\varepsilon}\left(x_{3}\right), \sup _{x_{3} \in[0,1]}\left|\tilde{\varrho}_{\varepsilon}\left(x_{3}\right)-1\right| \leq c \varepsilon^{2(m-n)} .
$$

As indicated by our choice of the initial data (1.7), the solutions of the evolutionary problem (1.1 - 1.31), (1.5), (1.7) satisfy far field conditions in the form

$$
\varrho \rightarrow \tilde{\varrho}_{\varepsilon}, \mathbf{u} \rightarrow 0 \text { as }|x| \rightarrow \infty .
$$

\section{$2.2 \quad$ Finite energy weak solutions}

We say that $[\varrho, \mathbf{u}]$ is a finite energy weak solution of the problem (1.1]-1.3), (1.5), (1.7), (2.6) on the space-time cylinder $(0, T) \times \Omega$ if the following holds:

- Regularity. The functions $\varrho$, u belong to the class

$$
\varrho \geq 0,\left(\varrho-\tilde{\varrho}_{\varepsilon}\right) \in L^{\infty}\left(0, T ; L^{2}+L^{\gamma}(\Omega)\right), \mathbf{u} \in L^{2}\left(0, T ; W^{1,2}\left(\Omega ; R^{3}\right)\right), \mathbf{u} \cdot \mathbf{n}=\left.u_{3}\right|_{\partial \Omega}=0 .
$$


- Equations. The equation of continuity (1.1) and the momentum equation (1.2) are replaced by integral identities

$$
\int_{0}^{T} \int_{\Omega}\left(\varrho \partial_{t} \varphi+\varrho \mathbf{u} \cdot \nabla_{x} \varphi\right) \mathrm{d} x \mathrm{~d} t=-\int_{\Omega} \varrho_{0, \varepsilon} \varphi(0, \cdot) \mathrm{d} x
$$

for any $\varphi \in C_{c}^{\infty}([0, T) \times \bar{\Omega})$, and

$$
\begin{aligned}
& \int_{0}^{T} \int_{\Omega}\left(\varrho_{\varepsilon} \mathbf{u}_{\varepsilon} \cdot \partial_{t} \varphi+(\varrho \mathbf{u} \otimes \mathbf{u}): \nabla_{x} \varphi-\frac{1}{\varepsilon} \varrho(\mathbf{f} \times \mathbf{u}) \cdot \varphi+\frac{1}{\varepsilon^{2 m}} p(\varrho) \operatorname{div}{ }_{x} \varphi\right) \mathrm{d} x \mathrm{~d} t \\
& =\int_{0}^{T} \int_{\Omega}\left(\varepsilon^{\alpha} \mathbb{S}\left(\nabla_{x} \mathbf{u}\right): \nabla_{x} \varphi-\frac{1}{\varepsilon^{2 n}} \varrho \nabla_{x} G \cdot \varphi\right) \mathrm{d} x \mathrm{~d} t-\int_{\Omega} \varrho_{0, \varepsilon} \mathbf{u}_{0, \varepsilon} \cdot \varphi(0, \cdot) \mathrm{d} x
\end{aligned}
$$

for any $\varphi \in C_{c}^{\infty}\left([0, T) \times \bar{\Omega} ; R^{3}\right),\left.\varphi \cdot \mathbf{n}\right|_{\partial \Omega}=0$.

- Energy. The energy inequality

$$
\begin{aligned}
\int_{\Omega}\left[\frac{1}{2} \varrho|\mathbf{u}|^{2}+\right. & \left.\frac{1}{\varepsilon^{2 m}}\left(H(\varrho)-H^{\prime}\left(\tilde{\varrho}_{\varepsilon}\right)\left(\varrho-\tilde{\varrho}_{\varepsilon}\right)-H\left(\tilde{\varrho}_{\varepsilon}\right)\right)\right](\tau, \cdot) \mathrm{d} x+\varepsilon^{\alpha} \int_{0}^{\tau} \int_{\Omega} \mathbb{S}\left(\nabla_{x} \mathbf{u}\right): \nabla_{x} \mathbf{u} \mathrm{d} x \mathrm{~d} t \\
& \leq \int_{\Omega}\left[\frac{1}{2} \varrho_{0, \varepsilon}\left|\mathbf{u}_{0, \varepsilon}\right|^{2}+\frac{1}{\varepsilon^{2 m}}\left(H\left(\varrho_{0, \varepsilon}\right)-H^{\prime}\left(\tilde{\varrho}_{\varepsilon}\right)\left(\varrho_{0, \varepsilon}-\tilde{\varrho}_{\varepsilon}\right)-H\left(\tilde{\varrho}_{\varepsilon}\right)\right)\right] \mathrm{d} x
\end{aligned}
$$

holds for a.a. $\tau \in(0, T)$.

Note that the existence theory in the class of finite energy weak solutions was developed by Lions [21] and later extended in [12] to the sofar "critical" adiabatic exponent $\gamma>\frac{3}{2}$.

\section{$2.3 \quad$ Relative entropy}

For future analysis, it is convenient to replace the energy inequality (2.10) by the relative entropy inequality containing more transparent piece of information on the asymptotic behavior of solutions for $\varepsilon \rightarrow 0$. To this end, we introduce the relative entropy functional

$$
\mathcal{E}_{\varepsilon}(\varrho, \mathbf{u} \mid r, \mathbf{U})=\int_{\Omega}\left[\frac{1}{2} \varrho|\mathbf{u}-\mathbf{U}|^{2}+\frac{1}{\varepsilon^{2 m}}\left(H(\varrho)-H^{\prime}(r)(\varrho-r)-H(r)\right)\right] \mathrm{d} x,
$$

cf. [10], [13], Germain [15].

It can be shown, see [10], that any finite energy weak solution $[\varrho, \mathbf{u}]$ specified in Section 2.2 satisfies the relative entropy inequality:

$$
\mathcal{E}_{\varepsilon}(\varrho, \mathbf{u} \mid r, \mathbf{U})(\tau)+\varepsilon^{\alpha} \int_{0}^{\tau} \int_{\Omega}\left(\mathbb{S}\left(\nabla_{x} \mathbf{u}\right)-\mathbb{S}\left(\nabla_{x} \mathbf{U}\right)\right):\left(\nabla_{x} \mathbf{u}-\nabla_{x} \mathbf{U}\right) \mathrm{d} x \mathrm{~d} t \leq
$$




$$
\begin{gathered}
\mathcal{E}_{\varepsilon}\left(\varrho_{0, \varepsilon}, \mathbf{u}_{0, \varepsilon} \mid r(0, \cdot), \mathbf{U}(0, \cdot)\right) \\
+\int_{0}^{\tau} \int_{\Omega} \varrho\left(\partial_{t} \mathbf{U}+\mathbf{u} \cdot \nabla_{x} \mathbf{U}\right) \cdot(\mathbf{U}-\mathbf{u}) \mathrm{d} x \mathrm{~d} t \\
+\varepsilon^{\alpha} \int_{0}^{\tau} \int_{\Omega} \mathbb{S}\left(\nabla_{x} \mathbf{U}\right): \nabla_{x}(\mathbf{U}-\mathbf{u}) \mathrm{d} x \mathrm{~d} t+\frac{1}{\varepsilon} \int_{0}^{\tau} \int_{\Omega} \varrho(\mathbf{f} \times \mathbf{u}) \cdot(\mathbf{U}-\mathbf{u}) \mathrm{d} x \mathrm{~d} t \\
+\frac{1}{\varepsilon^{2 m}} \int_{0}^{\tau} \int_{\Omega}\left[(r-\varrho) \partial_{t} H^{\prime}(r)+\nabla_{x}\left(H^{\prime}(r)-H^{\prime}\left(\varrho_{\varepsilon}\right)\right) \cdot(r \mathbf{U}-\varrho \mathbf{u})\right] \mathrm{d} x \mathrm{~d} t \\
-\frac{1}{\varepsilon^{2 m}} \int_{0}^{\tau} \int_{\Omega} \operatorname{div}_{x} \mathbf{U}(p(\varrho)-p(r)) \mathrm{d} x \mathrm{~d} t-\frac{1}{\varepsilon^{2 n}} \int_{0}^{\tau} \int_{\Omega}(\varrho-r) \nabla_{x} G \cdot \mathbf{U} \mathrm{d} x \mathrm{~d} t
\end{gathered}
$$

for all sufficiently smooth "test functions" $r$, U satisfying

$$
\left.\mathbf{U} \cdot \mathbf{n}\right|_{\partial \Omega}=0, r>0, \mathbf{U},\left(r-\tilde{\varrho}_{\varepsilon}\right) \text { compactly supported in } \bar{\Omega} \text {. }
$$

Note the assumptions concerning the decay and regularity can be relaxed to basically any couple $[r, \mathbf{U}]$ for which (2.12) makes sense via the standard density argument, see [10].

\section{Main result}

For a vector field $\mathbf{b}=\left[b_{1}, b_{2}, b_{2}\right]$, we introduce the horizontal component $\mathbf{b}_{h}=\left[b_{1}, b_{2}\right]$ writing $\mathbf{b}=$ $\left[\mathbf{b}_{h}, b_{3}\right]$. Similarly, we use the symbols $\nabla_{h}$, $\operatorname{div}_{h}$ to denote the differential operators acting on the horizontal variables only. Finally, the symbol $\mathbf{H}$ denotes the Helmholtz projection onto the space of solenoidal functions in $\Omega$, while $\mathbf{H}_{h}$ is the Helmholtz projection in $R^{2}$.

Let

$$
\mathbf{v}_{0} \in W^{k, 2}\left(R^{2} ; R^{2}\right), k \geq 3, \operatorname{div} \mathbf{v}_{0}=0,
$$

be given. It is well known (see for instance Kato and Lai [18]) that the Euler system (1.9) supplemented with the initial data

$$
\mathbf{v}(0)=\mathbf{v}_{0}
$$

admits a regular solution $(\mathbf{v}, \Pi)$, unique in the class

$$
\mathbf{v} \in C\left([0, T] ; W^{k, 2}\left(R^{2} ; R^{2}\right)\right), \partial_{t} \mathbf{v} \in C\left([0, T] ; W^{k-1,2}\left(R^{2} ; R^{2}\right)\right), \Pi \in C\left([0, T] ; W^{k, 2}\left(R^{2}\right)\right) .
$$

We are ready to formulate our main result. 
Theorem 3.1 Let the pressure $p=p(\varrho)$ satisfy the hypotheses (2.1), (2.2). Suppose that the exponents $\alpha, m, n$ are given such that

$$
\alpha>0, \frac{m}{2}>n \geq 1 .
$$

Let the initial data $\left[\varrho_{0, \varepsilon}, \mathbf{u}_{0, \varepsilon}\right]$ be given by (1.7), where the stationary states $\tilde{\varrho}_{\varepsilon}$ satisfy (2.4),

$$
\left\|\varrho_{0, \varepsilon}^{(1)}\right\|_{L^{2} \cap L^{\infty}(\Omega)} \leq c, \varrho_{0, \varepsilon}^{(1)} \rightarrow \varrho_{0}^{(1)} \text { in } L^{2}(\Omega), \mathbf{u}_{0, \varepsilon} \rightarrow \mathbf{u}_{0} \text { in } L^{2}\left(\Omega ; R^{3}\right),
$$

with

$$
\varrho_{0}^{(1)} \in W^{k-1,2}(\Omega), \mathbf{u}_{0} \in W^{k, 2}\left(\Omega ; R^{3}\right) \text { for a certain } k \geq 3 .
$$

Let $\left[\varrho_{\varepsilon}, \mathbf{u}_{\varepsilon}\right]$ be a finite energy weak solution of the problem (1.1 - 1.3), (1.5), (1.7), (2.6) in the space-time cylinder $(0, T) \times \Omega$.

Then

$$
\begin{gathered}
\text { ess } \sup _{t \in(0, T)}\left\|\varrho_{\varepsilon}(t, \cdot)-\tilde{\varrho}_{\varepsilon}\right\|_{\left(L^{2}+L^{\gamma}\right)(\Omega)} \leq \varepsilon^{m} c \\
\sqrt{\varrho_{\varepsilon}} \mathbf{u}_{\varepsilon} \rightarrow \mathbf{v}\left\{\begin{array}{l}
\text { weakly- }\left({ }^{*}\right) \text { in } L^{\infty}\left(0, T ; L^{2}\left(\Omega ; R^{3}\right)\right), \\
\text { strongly in } L_{\mathrm{loc}}^{1}\left((0, T) \times \Omega ; R^{3}\right),
\end{array}\right.
\end{gathered}
$$

where $\mathbf{v}=\left[\mathbf{v}_{h}, 0\right]$ is the unique solution of the Euler system (1.9), with the initial data

$$
\mathbf{v}(0, \cdot)=\mathbf{H}_{h}\left[\int_{0}^{1} \mathbf{u}_{0}\left(x_{h}, x_{3}\right) \mathrm{d} x_{3}\right] .
$$

The rest of the paper is devoted to the proof of Theorem 3.1.

\section{Uniform bounds}

We start with the nowadays standard estimates that follow directly from the energy inequality (2.10). These are conveniently formulated in terms of the essential and residual components of a measurable 
function $h$,

$$
\begin{gathered}
h=h_{\mathrm{ess}}+h_{\mathrm{res}}, \\
h_{\mathrm{ess}}=\chi\left(\varrho_{\varepsilon}\right) h, \chi \in C_{c}^{\infty}(0, \infty), 0 \leq \chi \leq 1, \chi=1 \text { in an open interval contaning } 1, \\
h_{\mathrm{res}}=\left(1-\chi\left(\varrho_{\varepsilon}\right)\right) h .
\end{gathered}
$$

Since the initial data are given by (1.7), where the functions $\varrho_{0, \varepsilon}^{(1)}, \mathbf{u}_{0, \varepsilon}$ satisfy the hypotheses (3.1), (3.2), the integral on the right-hand side of (2.2) is bounded uniformly for $\varepsilon \searrow 0$. As the stationary states $\tilde{\varrho}_{\varepsilon}$ are chosen to satisfy (2.5), we deduce the following bounds independent of $\varepsilon$ :

$$
\begin{gathered}
\operatorname{ess} \sup _{t \in(0, T)}\left\|\sqrt{\varrho_{\varepsilon}} \mathbf{u}_{\varepsilon}\right\|_{L^{2}\left(\Omega ; R^{3}\right)} \leq c, \\
\operatorname{ess} \sup _{t \in(0, T)}\left\|\left[\frac{\varrho_{\varepsilon}-\tilde{\varrho}_{\varepsilon}}{\varepsilon^{m}}\right]_{\operatorname{ess}}\right\|_{L^{2}(\Omega)} \leq c, \\
\operatorname{ess} \sup _{t \in(0, T)}\left\|\left[\varrho_{\varepsilon}\right]_{\mathrm{res}}\right\|_{L^{\gamma}(\Omega)}^{\gamma}+\operatorname{ess} \sup _{t \in(0, T)}\left\|[1]_{\mathrm{res}}\right\|_{L^{1}(\Omega)} \leq \varepsilon^{2 m} c,
\end{gathered}
$$

and

$$
\varepsilon^{\alpha} \int_{0}^{T} \int_{\Omega}\left|\nabla_{x} \mathbf{u}_{\varepsilon}+\nabla_{x} \mathbf{u}_{\varepsilon}^{t}-\frac{2}{3} \operatorname{div}_{x} \mathbf{u}_{\varepsilon} \mathbb{I}\right|^{2} \mathrm{~d} x \mathrm{~d} t \leq c
$$

cf. [9, Section 2].

Obviously, the estimates (4.1), (4.2) yield (3.3), which, combined with (2.5) gives rise to

$$
\varrho_{\varepsilon} \rightarrow 1 \text { in } L^{\infty}\left(0, T ; L_{\mathrm{loc}}^{q}(\Omega)\right) \text { for any } 1 \leq q<\gamma .
$$

Moreover, combining (4.5) with (4.1-4.3) we obtain

$$
\sqrt{\varrho_{\varepsilon}} \mathbf{u}_{\varepsilon} \rightarrow \mathbf{u} \text { weakly-(*) in } L^{\infty}\left(0, T ; L^{2}\left(\Omega ; R^{3}\right)\right),
$$

and

$$
\varrho_{\varepsilon} \mathbf{u}_{\varepsilon} \rightarrow \mathbf{u} \text { weakly- }\left(^{*}\right) \text { in } L^{\infty}\left(0, T ; L^{2}+L^{2 \gamma /(\gamma+1)}\left(\Omega ; R^{3}\right)\right),
$$

passing to suitable subsequences as the case may be.

Finally, we may let $\varepsilon \rightarrow 0$ in the equation of continuity to deduce that

$$
\operatorname{div}_{x} \mathbf{u}=0,\left.\mathbf{u} \cdot \mathbf{n}\right|_{\partial \Omega}=0 \text { in the sense of distributions in }(0, T) \times \Omega .
$$




\section{Decay estimates and oscillatory integrals}

With our convention (2.2), the equation describing the oscillatory part of solutions reads

$$
\begin{gathered}
\varepsilon^{m} \partial_{t} s+\operatorname{div}_{x} \mathbf{V}=0, \\
\varepsilon^{m} \partial_{t} \mathbf{V}+\omega \mathbf{f} \times \mathbf{V}+\nabla_{x} s=0, \omega=\varepsilon^{m-1},\left.\mathbf{V} \cdot \mathbf{n}\right|_{\partial \Omega}=0,
\end{gathered}
$$

cf. [11]. Re-scaling in the time we arrive at

$$
\begin{gathered}
\partial_{t} s+\operatorname{div}_{x} \mathbf{V}=0 \\
\partial_{t} \mathbf{V}+\omega \mathbf{b} \times \mathbf{V}+\nabla_{x} s=0,\left.\mathbf{V} \cdot \mathbf{n}\right|_{\partial \Omega}=0
\end{gathered}
$$

with the operator

$$
\mathcal{B}(\omega):\left[\begin{array}{c}
s \\
\mathbf{V}
\end{array}\right] \mapsto\left[\begin{array}{c}
\operatorname{div}_{x} \mathbf{V} \\
\omega \mathbf{f} \times \mathbf{V}+\nabla_{x} s
\end{array}\right] .
$$

The operator $\mathcal{B}$ is skew symmetric in the space $L^{2}(\Omega) \times L^{2}\left(\Omega ; R^{3}\right)$, with the domain of definition

$$
\mathcal{D}[\mathcal{B}(\omega)]=\left\{[r, \mathbf{V}]\left|r \in W^{1,2}(\Omega), \mathbf{V} \in L^{2}\left(\Omega ; R^{3}\right), \operatorname{div}_{x} \mathbf{V} \in L^{2}(\Omega), \mathbf{V} \cdot \mathbf{n}=V_{3}\right|_{\partial \Omega}=0\right\},
$$

and the kernel

$$
\mathcal{N}(\mathcal{B}(\omega))=\left\{[q, \mathbf{v}] \mid q=q\left(x_{h}\right), q \in W^{1,2}\left(R^{2}\right), \mathbf{v}=\left[\mathbf{v}_{h}\left(x_{h}\right), 0\right], \operatorname{div}_{h} \mathbf{v}_{h}=0, \omega \mathbf{f} \times \mathbf{v}+\nabla_{x} q=0\right\} .
$$

Let $\mathcal{P}(\omega)$ denote the projection

$$
\mathcal{P}(\omega): L^{2}(\Omega) \times L^{2}\left(\Omega ; R^{3}\right) \rightarrow \mathcal{N}(\mathcal{B}(\omega)) .
$$

Exactly as in [11, Section 4.1.1] we can show that

$$
\mathcal{P}(\omega)[r, \mathbf{U}]=[q, \mathbf{v}]
$$

if

$$
-\Delta_{h} q+\omega^{2} q=\omega \int_{0}^{1} \operatorname{curl}_{h} \mathbf{U}_{h} \mathrm{~d} x_{3}+\omega^{2} \int_{0}^{1} r \mathrm{~d} x_{3} \text { in } R^{2}, \mathbf{v}=\left[v_{1}, v_{2}, 0\right], \omega v_{1}=-\partial_{x_{2}} q, \omega v_{2}=\partial_{x_{1}} q .
$$




\section{$5.1 \quad$ Spectral analysis}

Thanks to our special choice of the geometry of the spatial domain $\Omega$, we may reformulate the problem (5.3), (5.4) in terms of the Fourier variables. To this end, we observe, exactly as in [11, that the underlying spatial domain $\Omega$ may be equivalently replaced by

$$
\tilde{\Omega}=R^{2} \times \mathcal{T}^{1}
$$

where

$$
\mathcal{T}^{1}=[-1,1]_{\{-1,1\}}
$$

is a "flat" sphere, and where $s, \mathbf{V}_{h}$ were extended as even functions of the vertical variable $x_{3}$, while $V_{3}$ was extended as odd in $x_{3}$. In other words, all quantities are understood as 2-periodic functions in the vertical $x_{3}$ variable.

Accordingly, for each function $g \in L^{2}(\tilde{\Omega})$, we introduce its Fourier representation

$$
\hat{g}(\xi, k), \quad \xi=\left[\xi_{1}, \xi_{2}\right] \in R^{2}, k \in Z
$$

where

$$
\hat{g}(\xi, k)=\frac{1}{\sqrt{2}} \int_{-1}^{1} \int_{R^{2}} \exp \left(-\mathrm{i} \xi \cdot x_{h}\right) g\left(x_{h}, x_{3}\right) \exp \left(-\mathrm{i} k x_{3}\right) \mathrm{d} x_{h} \mathrm{~d} x_{3} .
$$

We have

$$
g\left(x_{h}, x_{3}\right)=\sum_{k \in Z} \mathcal{F}_{\xi \rightarrow x_{h}}^{-1}[\hat{g}(\xi, k)] \exp \left(\mathrm{i} k x_{3}\right),
$$

where the symbol $\mathcal{F}_{x_{h} \rightarrow \xi}$ denotes the standard Fourier transform on $R^{2}$.

Thus the problem (5.3), (5.4) can be written in the form

$$
\frac{\mathrm{d}}{\mathrm{d} t}\left[\begin{array}{c}
\hat{s}(t, \xi, k) \\
\hat{V}_{1}(t, \xi, k) \\
\hat{V}_{2}(t, \xi, k) \\
\hat{V}_{3}(t, \xi, k)
\end{array}\right]+\mathrm{i}\left[\begin{array}{cccc}
0 & \xi_{1} & \xi_{2} & k \\
\xi_{1} & 0 & \omega \mathrm{i} & 0 \\
\xi_{2} & -\omega \mathrm{i} & 0 & 0 \\
k & 0 & 0 & 0
\end{array}\right]\left[\begin{array}{c}
\hat{s}(t, \xi, k) \\
\hat{V}_{1}(t, \xi, k) \\
\hat{V}_{2}(t, \xi, k) \\
\hat{V}_{3}(t, \xi, k)
\end{array}\right]=0,\left[\begin{array}{c}
\hat{s}(0, \xi, k) \\
\hat{\mathbf{V}}(0, \xi, k)
\end{array}\right]=\left[\begin{array}{c}
\hat{s}_{0}(\xi, k) \\
\hat{\mathbf{V}}_{0}(\xi, k)
\end{array}\right] ;
$$

with the Hermitian matrix

$$
\mathcal{A}(\xi, k, \omega)=\left[\begin{array}{cccc}
0 & \xi_{1} & \xi_{2} & k \\
\xi_{1} & 0 & \omega \mathrm{i} & 0 \\
\xi_{2} & -\omega \mathrm{i} & 0 & 0 \\
k & 0 & 0 & 0
\end{array}\right]
$$

Of course, solutions of (5.6) depend also on the parameter $\omega=\varepsilon^{m-1}$. 


\subsubsection{Spectral properties of the matrix $\mathcal{A}$}

After a bit tedious but straightforward manipulation (see [9]), we can check that $\mathcal{A}(\xi, k, \omega)$ possesses four eigenvalues

$$
\begin{aligned}
& \lambda_{1}\left(|\xi|^{2}, k, \omega\right)=\left[\frac{\omega^{2}+|\xi|^{2}+k^{2}+\sqrt{\left(\omega^{2}+|\xi|^{2}+k^{2}\right)^{2}-4 \omega^{2} k^{2}}}{2}\right]^{1 / 2}, \lambda_{2}\left(|\xi|^{2}, k, \omega\right)=-\lambda_{1}\left(|\xi|^{2}, k, \omega\right), \\
& \lambda_{3}\left(|\xi|^{2}, k, \omega\right)=\left[\frac{\omega^{2}+|\xi|^{2}+k^{2}-\sqrt{\left(\omega^{2}+|\xi|^{2}+k^{2}\right)^{2}-4 \omega^{2} k^{2}}}{2}\right]^{1 / 2}, \lambda_{4}\left(|\xi|^{2}, k, \omega\right)=-\lambda_{3}\left(|\xi|^{2}, k, \omega\right) .
\end{aligned}
$$

Note that $\lambda_{3}\left(|\xi|^{2}, 0, \omega\right)=\lambda_{4}\left(|\xi|^{2}, 0, \omega\right)=0$ are the zero eigenvalues corresponding to the non-void kernel of the matrix $\mathcal{A}$ - the Fourier image of the null-space of the operator $\mathcal{B}(\omega)$, see [9].

As for the eigenvectors $\left[q, v_{1}, v_{2}, v_{3}\right]$, we have

$$
\xi_{1} v_{1}+\xi_{2} v_{2}+k v_{3}=\lambda q, \xi_{1} q+\mathrm{i} \omega v_{2}=\lambda v_{1}, \xi_{2} q-\mathrm{i} \omega v_{1}=\lambda v_{2}, k q=\lambda v_{3}
$$

from which we immediately deduce

$$
v_{1}=\mu\left(\lambda \xi_{1}+\mathrm{i} \omega \xi_{2}\right), v_{2}=\mu\left(\lambda \xi_{2}-\mathrm{i} \omega \xi_{1}\right), \mu \lambda|\xi|^{2}=\lambda q-k v_{3}, \quad, k q=\lambda v_{3},
$$

where $\mu$ is a free parameter that is fixed to normalize the norm of the eigenvector to be one.

\subsubsection{Eigenvectors}

We denote by $\mathbf{E}=\mathbf{E}(\xi, k, \omega)=\left[q, v_{1}, v_{2}, v_{3}\right]$ the normalized eigenvectors. Our goal is to show that diagonalizing matrices $\mathcal{Q}, \mathcal{Q}^{T}$, formed by the eigenvectors, are $L^{p}$-multipliers in the $\xi$ variable restricted to compact subsets of $R^{2} \backslash\{0\}$. This amounts to showing that

$$
\sup _{\omega \in(0,1)} \max _{0<a \leq|\xi| \leq b<\infty}\left|\nabla_{\xi}^{A} E_{j}(\xi, k, \omega)\right| \leq c=c(A, a, b, k), j=1,2,3,4, k \in Z
$$

with any multi-index $A=\left(A_{1}, A_{2}\right)$.

We distinguish two cases:

Case $\lambda=0$ :

In this case, we necessarily have $k=0$ and $\lambda= \pm \lambda_{3}$, and the orthonormal basis of eigenvectors can be taken in the form

$$
\mathbf{E}_{1}=\mu\left[-\mathrm{i} \omega,-\xi_{2}, \xi_{1}, 0\right], \mu=\left(|\xi|^{2}+\omega^{2}\right)^{-1 / 2}, \mathbf{E}_{2}=[0,0,0,1]
$$

Clearly (5.10) holds. 
Case $\lambda \neq 0$ :

We find that

$$
v_{1}=\mu\left(\lambda \xi_{1}+\mathrm{i} \omega \xi_{2}\right), v_{2}=\mu\left(\lambda \xi_{2}-\mathrm{i} \omega \xi_{1}\right), \frac{\lambda^{2}-k^{2}}{\lambda^{2}} q=\mu\left(\xi_{1}^{2}+\xi_{2}^{2}\right), v_{3}=\frac{k}{\lambda} q .
$$

Thus, the corresponding normalized eigenvector has the form

$$
\mathbf{E}=\mu\left[\frac{\lambda^{2}|\xi|^{2}}{\lambda^{2}-k^{2}}, \lambda \xi_{1}+\mathrm{i} \omega \xi_{2}, \lambda \xi_{2}-\mathrm{i} \omega \xi_{1}, \frac{k \lambda|\xi|^{2}}{\lambda^{2}-k^{2}}\right]
$$

with

$$
\mu=\left[\frac{\lambda^{4}+|k|^{2} \lambda^{2}}{\left(\lambda^{2}-k^{2}\right)^{2}}|\xi|^{4}+\left(\lambda^{2}+\omega^{2}\right)|\xi|^{2}\right]^{-1 / 2} .
$$

We consider first the case $\lambda= \pm \lambda_{1}$. We check that

$$
\begin{gathered}
\lambda_{1}^{2} \geq|\xi|^{2} / 2, \quad\left(\omega^{2}+|\xi|^{2}+k^{2}\right)^{2}-4 \omega^{2} k^{2} \geq|\xi|^{4}, \\
\lambda_{1}^{2}-k^{2}=\frac{\omega^{2}-k^{2}+|\xi|^{2}+\sqrt{\left(\omega^{2}-k^{2}\right)^{2}+|\xi|^{2}\left(|\xi|^{2}+2\left(\omega^{2}+k^{2}\right)\right)}}{2} \geq \frac{|\xi|^{2}}{2} .
\end{gathered}
$$

Consequently (5.10) is satisfied.

Finally, if $0 \neq \lambda= \pm \lambda_{3}$, we note that

$$
\begin{gathered}
\lambda_{3}\left(|\xi|^{2}, k, \omega\right)=\omega|k| \frac{1}{\lambda_{1}\left(|\xi|^{2}, k, \omega\right)}, \quad \lambda_{3}^{2}-k^{2}=\frac{k^{2}\left(\omega^{2}-\lambda_{1}^{2}\right)}{\lambda_{1}^{2}}, \\
\lambda_{1}^{2}-\omega^{2}=\frac{-\omega^{2}+|\xi|^{2}+|k|^{2}+\sqrt{\left(\omega^{2}+|\xi|^{2}+k^{2}\right)^{2}-4 k^{2} \omega^{2}}}{2} \geq|\xi|^{2} / 2 .
\end{gathered}
$$

Using identity (5.12) $)_{1}$, we write $\mathbf{E}$ in terms of $\lambda_{1}$ and verify (5.10) employing this explicit formula and estimates (5.11), (5.12).

\subsection{Frequency cut-off}

As we shall see below, it will be convenient to approximate the initial data for the problem (5.3), (5.4) by a frequency truncation represented by a function $\psi \in C_{c}^{\infty}(0, \infty)$.

Accordingly, solutions of the problem (5.3), (5.4) will be, in a certain way, composed of the quantities

$$
Z\left(t, x_{h}, k, \omega\right)=\mathcal{F}_{\xi \rightarrow x_{h}}^{-1}\left[\exp \left( \pm \mathrm{i} \lambda_{j}\left(|\xi|^{2}, k, \omega\right) t\right) \psi(|\xi|) \hat{h}(\xi)\right], j=1,2,3,4, k \in Z, \omega \in(0,1),
$$


where $\hat{h}$ stands for the Fourier transform of the "initial data". Our goal will be to derive suitable dispersive estimates for the mapping $h \mapsto Z$.

We start with the $L^{1}-L^{\infty}$ decay estimates. To this end, write

$$
\|Z(t, \cdot, k, \omega)\|_{L^{\infty}\left(R^{2}\right)} \leq\left\|\mathcal{F}_{\xi \rightarrow x_{h}}^{-1}\left[\exp \left( \pm \mathrm{i} \lambda_{j}\left(|\xi|^{2}, k, \omega\right) t\right) \psi(|\xi|)\right]\right\|_{L^{\infty}\left(R^{2}\right)}\|h\|_{L^{1}\left(R^{2}\right)}
$$

Consequently,

$$
\begin{gathered}
\mathcal{F}_{\xi \rightarrow x_{h}}^{-1}\left[\exp \left( \pm \mathrm{i} \lambda_{j}\left(|\xi|^{2}, k, \omega\right) t\right) \psi(|\xi|)\right]\left(x_{h}\right) \\
=\pi \sqrt{2} \int_{0}^{2 \pi} \int_{0}^{\infty} \exp \left( \pm \mathrm{i} \lambda_{j}\left(|\xi|^{2}, k, \omega\right) t\right) \psi(|\xi|) \exp \left(\mathrm{i}|\xi|\left|x_{h}\right| \sin \theta\right)|\xi| \mathrm{d}|\xi| \mathrm{d} \theta \\
=\pi \sqrt{2} \int_{0}^{\infty} \exp \left( \pm \mathrm{i} \lambda_{j}\left(r^{2}, k, \omega\right) t\right) \psi(r) r J_{0}\left(r\left|x_{h}\right|\right) \mathrm{d} r
\end{gathered}
$$

where the symbols $J_{m}, m=0,1, \ldots$ denote the Bessel functions, cf. Guo, Peng, and Wang [16].

Finally, performing a simple change of variables, we get

$$
\mathcal{F}_{\xi \rightarrow x_{h}}^{-1}\left[\exp \left( \pm \mathrm{i} \lambda_{j}\left(|\xi|^{2}, k, \omega\right) t\right) \psi(|\xi|)\right]\left(x_{h}\right)=\frac{\pi \sqrt{2}}{2} \int_{0}^{\infty} \exp \left( \pm \mathrm{i} \lambda_{j}(z, k, \omega) t\right) \psi(\sqrt{z}) J_{0}\left(\sqrt{z}\left|x_{h}\right|\right) \mathrm{d} z .
$$

\subsection{Decay estimates}

Supposing $\lambda_{j} \neq 0$ we derive the desired decay estimates. To this end, we use van Corput's lemma, see Stein [30, Chapter 8.1.2, Proposition 2 and Corollary]:

Lemma 5.1 Let $\Lambda=\Lambda(z)$ be a smooth function away from the origin,

$$
\partial_{z} \Lambda(z) \text { monotone, }\left|\partial_{z} \Lambda(z)\right| \geq \Lambda_{0}>0
$$

for all $z \in[a, b], 0<a<b<\infty$. Let $\Phi$ be a smooth function on $[a, b]$.

Then

$$
\left|\int_{a}^{b} \exp (\mathrm{i} \Lambda(z) t) \Phi(z) \mathrm{d} z\right| \leq c \frac{1}{t \Lambda_{0}}\left[|\Phi(b)|+\int_{a}^{b}\left|\partial_{z} \Phi(z)\right| \mathrm{d} z\right],
$$

where $c$ is an absolute constant independent of $\Lambda$ and $\Phi$. 
Going back to the oscillatory integral (5.16), we distinguish two cases.

\subsubsection{Case $\left|x_{h}\right|>t^{\beta}$}

Using the decay properties of $J_{0}$ and the fact that $\psi$ is compactly supported away from zero, we get

$$
\left|\mathcal{F}_{\xi \rightarrow x_{h}}^{-1}\left[\exp \left( \pm \mathrm{i} \lambda_{j}\left(|\xi|^{2}, k, \omega\right) t\right) \psi(|\xi|)\right]\left(x_{h}\right)\right| \leq c(\psi) t^{-\beta / 2} \text { whenever }\left|x_{h}\right|>t^{\beta}
$$

\subsubsection{Case $\left|x_{h}\right| \leq t^{\beta}$}

The idea is to use van Corput's lemma. Let $[a, b]$ be a closed interval, $a>0$, containing the support of $\psi(\sqrt{z})$. In accordance with the hypotheses of Lemma 5.1, we have to verify that

$$
\left|\partial_{z} \lambda_{j}(z, k, \omega)\right| \geq \Lambda_{0}(a, b, \omega, k)>0 \text { for all } z \in[a, b], \omega \in(0,1)
$$

- $\partial_{z} \Lambda_{j}$ is monotone on $[a, b]$.

If the two conditions are satisfied, we get

$$
\left|\mathcal{F}_{\xi \rightarrow x_{h}}^{-1}\left[\exp \left( \pm \mathrm{i} \lambda_{j}\left(|\xi|^{2}, k, \omega\right) t\right) \psi(|\xi|)\right]\left(x_{h}\right)\right| \leq c(\psi) \frac{1}{\Lambda_{0}(a, b, k, \omega) t}\left(1+t^{\beta / 2}\right) \text { for }\left|x_{h}\right| \leq t^{\beta}
$$

where again we have used the properties of the Bessel functions, namely,

$$
J^{\prime}{ }_{0}(z)=-J_{1}(z) .
$$

Now, our goal is to verify the hypotheses of Lemma 5.1. We have

$$
\partial_{z} \lambda_{1}(z, k, \omega)=\frac{1}{2} \frac{1}{\lambda_{1}(z, k, \omega)} \beta_{1}(z, k, \omega), \beta_{1}(z, k, \omega)=\frac{1}{2}\left(1+\frac{\omega^{2}+z+k^{2}}{\sqrt{\left(\omega^{2}+z+k^{2}\right)^{2}-4 \omega^{2} k^{2}}}\right),
$$

and, similarly,

$$
\partial_{z} \lambda_{3}(z, k, \omega)=\frac{1}{2} \frac{1}{\lambda_{3}(z, k, \omega)} \beta_{3}(z, k, \omega), \beta_{3}(z, k, \omega)=\frac{1}{2}\left(1-\frac{\omega^{2}+z+k^{2}}{\sqrt{\left(\omega^{2}+z+k^{2}\right)^{2}-4 \omega^{2} k^{2}}}\right), k \neq 0 .
$$

Furthermore,

$$
\partial_{z} \beta_{1}(z, k, \omega)=-\frac{2 \omega^{2} k^{2}}{\left[\left(\omega^{2}+z+k^{2}\right)^{2}-4 \omega^{2} k^{2}\right]^{3 / 2}}, \partial_{z} \beta_{3}(z, k, \omega)=\frac{2 \omega^{2} k^{2}}{\left[\left(\omega^{2}+z+k^{2}\right)^{2}-4 \omega^{2} k^{2}\right]^{3 / 2}} .
$$


Summing up the previous relations, we conclude that

$$
\partial_{z} \lambda_{1}(z, k, \omega) \text { is a decreasing functions of } z
$$

while

$$
\partial_{z} \lambda_{3}(z, k, \omega) \text { is an increasing functions of } z \text { for } k \neq 0 .
$$

Consequently, we deduce that

$$
\partial_{z} \lambda_{1}(z, k, \omega) \geq \partial_{z} \Lambda_{1}(b, k, \omega) \geq \Lambda(\psi, k) \text { for } z \in[a, b], \omega \in(0,1) .
$$

Finally, we have

$$
\partial_{z} \lambda_{3}(z, k, \omega)<0,\left|\partial_{z} \lambda_{3}(z, k, \omega)\right| \geq\left|\partial_{z} \lambda_{3}(b, k, \omega)\right|,
$$

where

$$
\begin{gathered}
\left|\partial_{z} \lambda_{3}(b, k, \omega)\right| \geq \frac{1}{2}\left(\frac{\omega^{2}+b+k^{2}}{\sqrt{\left(\omega^{2}+b+k^{2}\right)^{2}-4 \omega^{2} k^{2}}}-1\right)\left(\frac{\omega^{2}+b+k^{2}-\sqrt{\left(\omega^{2}+b+k^{2}\right)^{2}-4 \omega^{2} k^{2}}}{2}\right)^{-1 / 2} \\
=\sqrt{2} \omega|k|\left(\left(\omega^{2}+b+k^{2}\right)^{2}-4 \omega^{2} k^{2}\right)^{-1 / 2}\left(\omega^{2}+b+k^{2}+\sqrt{\left(\omega^{2}+b+k^{2}\right)^{2}-4 \omega^{2} k^{2}}\right)^{-1 / 2} \\
\geq c(\psi, k) \omega \text { for } k \neq 0, \omega \in(0,1) .
\end{gathered}
$$

Thus, reviewing (5.17), (5.18) we may infer that

$$
\left\|\mathcal{F}_{\xi \rightarrow x_{h}}^{-1}\left[\exp \left( \pm \mathrm{i} \lambda_{j}\left(|\xi|^{2}, k, \omega\right) t\right) \psi(|\xi|)\right]\right\|_{L^{\infty}\left(R^{2}\right)} \leq c(\psi, k) \max \left\{\frac{1}{\omega t^{1-\beta / 2}} ; \frac{1}{t^{\beta / 2}}\right\}, t>0
$$

as soon as $\lambda_{j} \neq 0$, which gives rise to the decay estimates

$$
\|Z(t, \cdot, k, \omega)\|_{L^{\infty}\left(R^{2}\right)} \leq c(\psi, k) \max \left\{\frac{1}{\omega t^{1-\beta / 2}} ; \frac{1}{t^{\beta / 2}}\right\}\|h\|_{L^{1}\left(R^{2}\right)} .
$$

Next, seeing that the mapping $h \mapsto \exp \left(\mathrm{i} \lambda_{j}(\xi, k, \omega) t\right) h$ is an isometry on $L^{2}\left(R^{2}\right)$, we deduce

$$
\|Z(t, \cdot, k, \omega)\|_{L^{2}\left(R^{2}\right)} \leq c\|h\|_{L^{2}\left(R^{2}\right)}
$$

Finally, interpolating (5.21) and (5.22), we obtain the $L^{p}-L^{q}$ estimates

$$
\|Z(t, \cdot, k, \omega)\|_{L^{p}\left(R^{2}\right)} \leq c(\psi, p, k) \max \left\{\frac{1}{\omega t^{1-\beta / 2}} ; \frac{1}{t^{\beta / 2}}\right\}^{1-\frac{2}{p}}\|h\|_{L^{p^{\prime}\left(R^{2}\right)}} \text { for } p \geq 2, \frac{1}{p}+\frac{1}{p^{\prime}}=1, \lambda_{j} \neq 0 .
$$


Keeping in mind that $\omega$ scales like $\varepsilon^{m-1}$ while the time $t$ is proportional to $\varepsilon^{-m}$ we observe that taking

$$
0<\beta<\frac{2}{m}
$$

yields the effective decay of $Z_{\varepsilon}=Z\left(t / \varepsilon^{m}, k, \omega\right)$ on any compact subinterval of $(0, T]$. In particular, the optimal choice $\beta=1 / m$ gives rise to

$$
\left\|Z\left(\frac{t}{\varepsilon^{m}}, \cdot, k, \omega\right)\right\|_{L^{p}\left(R^{2}\right)} \leq c \varepsilon^{\frac{1}{2}-\frac{1}{p}} \max \left\{\frac{1}{t^{1-1 / 2 m}} ; \frac{1}{t^{1 / 2 m}}\right\}^{1-\frac{2}{p}}\|h\|_{L^{p^{\prime}\left(R^{2}\right)}}, p \geq 2, \lambda_{j} \neq 0, t \in(0, T] .
$$

\section{Convergence}

In this final part, we complete the proof of Theorem 3.1. The basic idea is to use the relative entropy inequality (2.12) for a suitable choice of test functions $r$ and $\mathbf{U}$.

\subsection{Initial data decomposition}

We start be introducing suitable smoothing operators imposed on the initial data. Taking a family of smooth functions

$$
\psi_{\delta} \in C_{c}^{\infty}(0, \infty), 0 \leq \psi_{\delta} \leq 1, \psi_{\delta} \nearrow 1 \text { as } \delta \rightarrow 0
$$

and

$$
\phi_{\delta}=\phi_{\delta}\left(x_{h}\right) \in C_{c}^{\infty}\left(R^{2}\right), 0 \leq \phi_{\delta} \leq 1, \phi_{\delta} \nearrow 1 \text { as } \delta \rightarrow 0,
$$

we introduce

$$
\left[\varrho_{0}^{(1)}\right]_{\delta}\left(x_{h}, x_{3}\right)=\frac{1}{\sqrt{2}} \sum_{|k| \leq 1 / \delta} \mathcal{F}_{\xi \rightarrow x_{h}}^{-1}\left[\psi_{\delta}(|\xi|)\left(\varrho_{0}^{\widehat{(1)}} \phi_{\delta}\right)(\xi, k)\right] \exp \left(-\mathrm{i} k x_{3}\right)
$$

and, similarly,

$$
\left[u_{0, j}\right]_{\delta}\left(x_{h}, x_{3}\right)=\frac{1}{\sqrt{2}} \sum_{|k| \leq 1 / \delta} \mathcal{F}_{\xi \rightarrow x_{h}}^{-1}\left[\psi_{\delta}(|\xi|)\left(\widehat{u_{0, j} \phi_{\delta}}\right)(\xi, k)\right] \exp \left(-\mathrm{i} k x_{3}\right), j=1,2,3 .
$$

Now, we write the initial data in the form

$$
\begin{gathered}
{\left[\varrho_{0}^{(1)}\right]_{\delta}=s_{0, \varepsilon, \delta}+q_{0, \varepsilon, \delta}, \text { where }-\Delta_{h} q_{0, \varepsilon, \delta}+\omega^{2} q_{0, \delta}=\omega \int_{0}^{1} \operatorname{curl}_{h}\left[\left[\mathbf{u}_{0}\right]_{h}\right]_{\delta} \mathrm{d} x_{3}+\omega^{2} \int_{0}^{1}\left[\varrho_{0}^{(1)}\right]_{\delta} \mathrm{d} x_{3}} \\
{\left[\mathbf{u}_{0}\right]_{\delta}=\mathbf{V}_{0, \varepsilon, \delta}+\mathbf{v}_{0, \varepsilon, \delta}, \text { with } \omega\left[v_{0, \varepsilon, \delta}\right]_{1}=-\partial_{x_{2}} q_{0, \varepsilon, \delta}, \omega\left[v_{0, \varepsilon, \delta}\right]_{2}=\partial_{x_{1}} q_{0, \varepsilon, \delta}}
\end{gathered}
$$


Finally, we choose the functions $r, \mathbf{U}$ in the relative entropy inequality as

$$
r=r_{\varepsilon, \delta}=\tilde{\varrho}_{\varepsilon}+\varepsilon^{m}\left(q_{\varepsilon, \delta}+s_{\varepsilon, \delta}\right), \mathbf{U}=\mathbf{U}_{\varepsilon, \delta}=\mathbf{v}_{\varepsilon, \delta}+\mathbf{V}_{\varepsilon, \delta},
$$

where $\left[s_{\varepsilon, \delta}, \mathbf{V}_{\varepsilon, \delta}\right]$ is the unique solution of the acoustic-Rossby system (5.1), (5.2), emanating from the initial data

$$
s_{\varepsilon, \delta}(0, \cdot)=s_{0, \varepsilon, \delta}, \quad \mathbf{V}_{\varepsilon, \delta}(0, \cdot)=\mathbf{V}_{0, \varepsilon, \delta},
$$

while the functions $q_{\varepsilon, \delta}, \mathbf{v}_{\varepsilon, \delta}$ are interrelated through

$$
\omega \mathbf{f} \times \mathbf{v}_{\varepsilon, \delta}+\nabla_{x} q_{\varepsilon, \delta}=0,
$$

where $q_{\varepsilon, \delta}$ is the unique solution of the problem

$$
\partial_{t}\left(\Delta_{h} q_{\varepsilon, \delta}-\omega^{2} q_{\varepsilon, \delta}\right)+\frac{1}{\omega} \nabla_{h}^{\perp} q_{\varepsilon, \delta} \cdot \nabla_{x}\left(\Delta_{h} q_{\varepsilon, \delta}-\omega^{2} q_{\varepsilon, \delta}\right)=0, q_{\varepsilon, \delta}(0, \cdot)=q_{0, \varepsilon, \delta} .
$$

\subsection{Decay of the oscillatory component}

First we claim that, in view of the dispersive estimates (5.24) (with $0<\beta<2 / m$ ), and the properties of the eigenvectors of the matrix $\mathcal{A}$, discussed in detail in Section [5.1.2, we get

$$
s_{\varepsilon, \delta} \rightarrow 0 \text { in } L^{p}\left(0, T ; W^{l, q}(\Omega)\right), \mathbf{V}_{\varepsilon, \delta} \rightarrow 0 \text { in } L^{p}\left(0, T ; W^{l, q}(\Omega)\right) \text { as } \varepsilon \rightarrow 0
$$

for any fixed $\delta>0,1 \leq p<\infty, 2<q \leq \infty$ and $l=0,1, \ldots$

We note that

$$
\begin{aligned}
& {\left[\begin{array}{c}
s_{\varepsilon, \delta}\left(t, x_{h}, k\right) \\
\mathbf{V}_{\varepsilon, \delta}\left(t, x_{h}, k\right.
\end{array}\right]=\mathcal{F}_{\xi \rightarrow x_{h}}^{-1}\left[\mathcal{Q}^{T}(\xi, \omega, k)\left[\begin{array}{c}
\exp \left(\mathrm{i} \lambda_{1}(\xi, \omega, k) \frac{t}{\varepsilon^{m}}\right), 0,0,0 \\
0, \exp \left(\mathrm{i} \lambda_{2}(\xi, \omega, k) \frac{t}{\varepsilon^{m}}\right), 0,0 \\
0,0, \exp \left(\mathrm{i} \lambda_{3}(\xi, \omega, k) \frac{t}{\varepsilon^{m}}\right), 0 \\
0,0,0, \exp \left(\mathrm{i} \lambda_{4}(\xi, \omega, k) \frac{t}{\varepsilon^{m}}\right)
\end{array}\right] \mathcal{Q}(\xi, \omega, k) \psi(|\xi|) \hat{h}_{0}(\xi)\right]} \\
& =\mathcal{F}_{\xi \rightarrow x_{h}}^{-1}\left[\tilde{\psi}(|\xi|) \mathcal{Q}^{T}(\xi, \omega, k)\left[\begin{array}{c}
\exp \left(\mathrm{i} \lambda_{1}(\xi, \omega, k) \frac{t}{\varepsilon^{m}}\right), 0,0,0 \\
0, \exp \left(\mathrm{i} \lambda_{2}(\xi, \omega, k) \frac{t}{\varepsilon^{m}}\right), 0,0 \\
0,0, \exp \left(\mathrm{i} \lambda_{3}(\xi, \omega, k) \frac{t}{\varepsilon^{m}}\right), 0 \\
0,0,0, \exp \left(\mathrm{i} \lambda_{4}(\xi, \omega, k) \frac{t}{\varepsilon^{m}}\right)
\end{array}\right] \tilde{\psi}(|\xi|) \mathcal{Q}(\xi, \omega, k) \psi(|\xi|) \hat{h}_{0}(\xi)\right], \omega=\varepsilon^{m-1},
\end{aligned}
$$


where $\tilde{\psi} \in C_{c}^{\infty}(0, \infty)$ has been chosen so that $\psi \tilde{\psi}=\psi$.

In accordance with Section 5.1.2, the quantities $\tilde{\psi} \mathcal{Q}, \tilde{\psi} \mathcal{Q}^{T}$ are $L^{p}-$ Fourier multipliers with norm independent of $\omega$. Thus the desired decay estimates (6.8) follow from (5.24).

\subsection{Convergence of the non-oscillatory component}

We introduce the scaled function $\tilde{q}_{\varepsilon, \delta}=q_{\varepsilon, \delta} / \omega$ and observe that

$$
\begin{gathered}
\partial_{t}\left(\Delta_{h} \tilde{q}_{\varepsilon, \delta}-\omega^{2} \tilde{q}_{\varepsilon, \delta}\right)+\nabla_{h}^{\perp} \tilde{q}_{\varepsilon, \delta} \cdot \nabla_{h}\left(\Delta_{h} \tilde{q}_{\varepsilon, \delta}-\omega^{2} \tilde{q}_{\varepsilon, \delta}\right), q_{\varepsilon, \delta}(0)=q_{0, \varepsilon, \delta} \\
\Delta_{h} \tilde{q}_{0, \varepsilon, \delta}-\omega^{2} \tilde{q}_{0, \delta}=\int_{0}^{1} \operatorname{curl}_{h}\left[\left[\mathbf{u}_{0}\right]_{h}\right]_{\delta} \mathrm{d} x_{3}+\omega \int_{0}^{1}\left[\varrho_{0}^{(1)}\right]_{\delta} \mathrm{d} x_{3} .
\end{gathered}
$$

Since the initial data are regular, we may use the result of Oliver [26, Theorem 3] to deduce that

$$
\left\{\Delta \tilde{q}_{\varepsilon, \delta}+\varepsilon^{2} \tilde{q}_{\varepsilon, \delta}\right\}_{\varepsilon>0} \text { is bounded in } C^{r}\left([0, T] ; W^{l, 2}(\Omega)\right), r \geq 0, l=0,1, \ldots
$$

Moreover, as $\Delta \tilde{q}_{\varepsilon, \delta}$ satisfies that transport equation (6.9) with the initial data in $L^{p}\left(R^{2}\right)$, we get

$$
\left\{\Delta \tilde{q}_{\varepsilon, \delta}+\omega^{2} \tilde{q}_{\varepsilon, \delta}\right\}_{\varepsilon>0} \text { is bounded in } L^{\infty}\left([0, T] ; L^{p}(\Omega)\right) \text { for any } 1<p<\infty .
$$

Next, we recall the "energy estimates" that can be obtained multiplying ([6.9) on $\tilde{q}_{\varepsilon, \delta}$ and integrating by parts:

$$
\int_{R^{2}}\left(\left|\nabla_{x} \tilde{q}_{\varepsilon, \delta}\right|^{2}+\omega^{2}\left|\tilde{q}_{\varepsilon, \delta}\right|^{2}\right)(\tau, \cdot) \mathrm{d} x=\int_{R^{2}}\left(\left|\nabla_{x} \tilde{q}_{0, \varepsilon, \delta}\right|^{2}+\omega^{2}\left|\tilde{q}_{0, \varepsilon, \delta}\right|^{2}\right) \mathrm{d} x .
$$

Note that

$$
\int_{R^{2}} \nabla_{h}^{\perp} \tilde{q}_{\varepsilon, \delta} \cdot \nabla_{h} \Delta_{h} \tilde{q}_{\varepsilon, \delta} \tilde{q}_{\varepsilon, \delta} \mathrm{d} x=-\int_{R^{2}} \nabla_{h}^{\perp} \tilde{q}_{\varepsilon, \delta} \cdot \nabla_{h} \tilde{q}_{\varepsilon, \delta} \Delta_{h} \tilde{q}_{\varepsilon, \delta} \mathrm{d} x=0
$$

Since

$$
\mathbf{v}_{\varepsilon, \delta}=\nabla_{h}^{\perp} \tilde{q}_{\varepsilon, \delta}
$$

we get

$$
\left\{\mathbf{v}_{\varepsilon, \delta}\right\}_{\varepsilon>0} \text { is bounded in } C^{r}\left([0, T] ; W^{l, 2}\left(R^{2}\right)\right), r \geq 0, l=0,1, \ldots
$$

Finally, we compute

$$
\partial_{t} \tilde{q}_{\varepsilon, \delta}=\left(\Delta_{h}-\omega^{2}\right)^{-1}\left[\nabla_{h}\left(\mathbf{v}_{\varepsilon, \delta} \operatorname{curl} \mathbf{v}_{\varepsilon, \delta}\right)\right]
$$

where, furthermore,

$$
\operatorname{vcurl}_{h} \mathbf{v}=\left[v_{1}\left(\partial_{x_{1}} v_{2}-\partial_{x_{2}} v_{1}\right) ; v_{2}\left(\partial_{x_{1}} v_{2}-\partial_{x_{2}} v_{1}\right)\right]
$$

with

$$
v_{1} \partial_{x_{1}} v_{2}=\partial_{x_{1}}\left(v_{1} v_{2}\right)+\frac{1}{2} \partial_{x_{2}} v_{2}^{2}, v_{2} \partial_{x_{2}} v_{1}=\partial_{x_{2}}\left(v_{1} v_{2}\right)+\frac{1}{2} \partial_{x_{1}} v_{1}^{2}
$$


Consequently, we may infer that

$$
\left\{\partial_{t} \tilde{q}_{\varepsilon, \delta}\right\}_{\varepsilon>0} \text { is bounded in } C^{r}\left([0, T] ; W^{l, q}\left(R^{2}\right)\right), r \geq 0, q>1, l=0,1, \ldots
$$

All the above estimates may depend on $\delta$ but are uniform with respect to $\varepsilon \searrow 0$.

In view of the above estimates, it is easy to pass to the limit for $\varepsilon \rightarrow 0$ in order to get

$$
\mathbf{v}_{\varepsilon, \delta} \rightarrow \mathbf{v}_{\delta}, \partial_{t} \mathbf{v}_{\varepsilon, \delta} \rightarrow \partial_{t} \mathbf{v}_{\delta} \text { weakly-(*) in } L^{\infty}\left(0, T ; W^{l, 2}\left(R^{2}\right)\right), l=0,1, \ldots,
$$

where

$$
\mathbf{v}_{\delta}, \partial_{t} \mathbf{v}_{\delta} \in C\left([0, T] ; W^{l, 2}\left(R^{2}\right)\right), l=0,1,2, \ldots
$$

We have, in particular,

$$
\mathbf{v}_{\varepsilon, \delta} \rightarrow \mathbf{v}_{\delta} \text { in } L^{q}\left(0, T ; L_{\mathrm{loc}}^{q}(\Omega)\right), 1 \leq q<\infty ;
$$

whence, by virtue of (6.9),

$$
\partial_{t} \operatorname{curl}_{h} \mathbf{v}_{\delta}+\mathbf{v}_{\delta} \cdot \nabla_{h} \operatorname{curl}_{h} \mathbf{v}_{\delta}=0 .
$$

Seeing that

$$
\operatorname{curl}_{h} \operatorname{div}_{h}\left(\mathbf{v}_{\delta} \otimes \mathbf{v}_{\delta}\right)=\operatorname{curl}_{h}\left(\mathbf{v}_{\delta} \cdot \nabla_{h} \mathbf{v}_{\delta}\right)=\mathbf{v}_{\delta} \cdot \nabla_{h} \operatorname{curl}_{h} \mathbf{v}_{\delta}
$$

we deduce existence of

$$
\Pi_{\delta} \in C\left([0, T] ; W^{l, 2}\left(R^{2}\right)\right), l=0,1,2, \ldots,
$$

where the couple $\left(\mathbf{v}_{\delta}, \Pi_{\delta}\right)$ is the unique solution of the Euler system (1.9), emanating from the initial data

$$
\mathbf{v}_{\delta}(0, \cdot)=\int_{0}^{1} \mathbf{H}_{h}\left[\left[\mathbf{u}_{0}\right]_{\delta}\right] \mathrm{d} x_{3} .
$$

\subsection{Relative entropy inequality}

We return to the relative entropy inequality (2.12), where $\varrho=\varrho_{\varepsilon}, \mathbf{u}=\mathbf{u}_{\varepsilon}$ and the test functions $r$ and $\mathbf{U}$ are given by (6.5). In what follows, we examine step by step all terms on the right-hand side of (2.12) and perform the limits; first for $\varepsilon \rightarrow 0$, then for $\delta \rightarrow 0$. 


\subsubsection{Initial data}

We have

$$
\begin{gathered}
\mathcal{E}_{\varepsilon}\left(\varrho_{0, \varepsilon}, \mathbf{u}_{0, \varepsilon} \mid r(0, \cdot), \mathbf{U}(0, \cdot)\right) \\
=\int_{\Omega} \frac{1}{2} \varrho_{0, \varepsilon}\left|\mathbf{u}_{0, \varepsilon}-\left[\mathbf{u}_{0}\right]_{\delta}\right|^{2} \mathrm{~d} x \\
+\int_{\Omega}\left[\frac{1}{\varepsilon^{2 m}}\left(H\left(1+\varepsilon^{m} \varrho_{0, \varepsilon}^{(1)}\right)-H^{\prime}\left(1+\varepsilon^{m}\left[\varrho_{0}^{(1)}\right]_{\delta}\right)\left(\left(\varrho_{0, \varepsilon}^{(1)}-\left[\varrho_{0}^{(1)}\right]_{\delta}\right)-H\left(1+\varepsilon^{m}\left[\varrho_{0}^{(1)}\right]_{\delta}\right)\right)\right] \mathrm{d} x\right. \\
\leq c\left(\left\|\mathbf{u}_{0, \varepsilon}-\left[\mathbf{u}_{0}\right]_{\delta}\right\|_{L^{2}\left(\Omega ; R^{3}\right)}^{2}+\left\|\varrho_{0, \varepsilon}^{(1)}-\left[\varrho_{0}^{(1)}\right]_{\delta}\right\|_{L^{2}\left(\Omega ; R^{3}\right)}^{2}\right) \\
\rightarrow c\left(\left\|\mathbf{u}_{0}-\left[\mathbf{u}_{0}\right]_{\delta}\right\|_{L^{2}\left(\Omega ; R^{3}\right)}^{2}+\left\|\varrho_{0}^{(1)}-\left[\varrho_{0}^{(1)}\right]_{\delta}\right\|_{L^{2}\left(\Omega ; R^{3}\right)}^{2}\right) \text { as } \varepsilon \rightarrow 0 .
\end{gathered}
$$

The most left quantity obviously tends to zero as $\delta \searrow 0$.

\subsubsection{Viscosity}

We write

$$
\varepsilon^{\alpha}\left|\int_{0}^{\tau} \int_{\Omega} \mathbb{S}\left(\nabla_{x} \mathbf{U}_{\varepsilon, \delta}\right): \nabla_{x}\left(\mathbf{U}_{\varepsilon, \delta}-\mathbf{u}_{\varepsilon}\right) \mathrm{d} x \mathrm{~d} t\right| \leq \varepsilon^{\alpha} c_{1}(\delta) \int_{0}^{\tau} \int_{\Omega}\left|\nabla_{x}\left(\mathbf{U}_{\varepsilon, \delta}-\mathbf{u}_{\varepsilon}\right)\right| \mathrm{d} x \mathrm{~d} t,
$$

and, by Korn's inequality,

$$
\varepsilon^{\alpha} \int_{0}^{\tau} \int_{\Omega}\left|\nabla_{x}\left(\mathbf{U}_{\varepsilon, \delta}-\mathbf{u}_{\varepsilon}\right)\right| \mathrm{d} x \mathrm{~d} t \leq \frac{\varepsilon^{\alpha}}{2} \int_{0}^{\tau} \int_{\Omega}\left(\mathbb{S}_{\varepsilon}\left(\nabla_{x} \mathbf{u}_{\varepsilon}\right)-\mathbb{S}_{\varepsilon}\left(\nabla_{x} \mathbf{U}_{\varepsilon, \delta}\right): \nabla_{x}\left(\mathbf{U}_{\varepsilon, \delta}-\mathbf{u}_{\varepsilon}\right) \mathrm{d} x \mathrm{~d} t+c_{2} \varepsilon^{\alpha} .\right.
$$

\subsubsection{Forcing term}

We have

$$
\begin{gathered}
\frac{1}{\varepsilon^{2 m}}\left[\left(r_{\varepsilon, \delta}-\varrho_{\varepsilon}\right) \partial_{t} H^{\prime}\left(r_{\varepsilon, \delta}\right)+\nabla_{x}\left(H^{\prime}\left(r_{\varepsilon, \delta}\right)-H^{\prime}\left(\varrho_{\varepsilon}\right)\right) \cdot\left(r_{\varepsilon, \delta} \mathbf{U}_{\varepsilon, \delta}-\varrho_{\varepsilon} \mathbf{u}_{\varepsilon}\right)\right] \\
+\frac{1}{\varepsilon^{2 m}} \operatorname{div}_{x} \mathbf{U}_{\varepsilon, \delta}\left(p\left(r_{\varepsilon, \delta}\right)-p\left(\varrho_{\varepsilon}\right)\right)+\frac{1}{\varepsilon^{2 n}}\left(r_{\varepsilon, \delta}-\varrho_{\varepsilon}\right) \nabla_{x} G \cdot \mathbf{U}_{\varepsilon, \delta} \\
=\frac{1}{\varepsilon^{2 m}}\left[p\left(r_{\varepsilon, \delta}\right)-p^{\prime}\left(r_{\varepsilon, \delta}\right)\left(r_{\varepsilon, \delta}-\varrho_{\varepsilon}\right)-p\left(\varrho_{\varepsilon}\right)\right] \operatorname{div}_{x} \mathbf{U}_{\varepsilon, \delta}+\frac{1}{\varepsilon^{2 m}}\left(r_{\varepsilon, \delta}-\varrho_{\varepsilon}\right) H^{\prime \prime}\left(r_{\varepsilon, \delta}\right)\left[\partial_{t} r_{\varepsilon, \delta}+\operatorname{div}_{x}\left(r_{\varepsilon, \delta} \mathbf{U}_{\varepsilon, \delta}\right)\right] \\
+\frac{1}{\varepsilon^{2 m}}\left[\varrho_{\varepsilon} \nabla_{x} H^{\prime}\left(r_{\varepsilon, \delta}\right) \cdot\left(\mathbf{U}_{\varepsilon, \delta}-\mathbf{u}_{\varepsilon}\right)-\nabla_{x} H^{\prime}\left(\varrho_{\varepsilon}\right) \cdot\left(r_{\varepsilon, \delta} \mathbf{U}_{\varepsilon, \delta}-\varrho_{\varepsilon} \mathbf{u}_{\varepsilon}\right)\right]+\frac{1}{\varepsilon^{2 n}}\left(r_{\varepsilon, \delta}-\varrho_{\varepsilon}\right) \nabla_{x} G \cdot \mathbf{U}_{\varepsilon, \delta},
\end{gathered}
$$

where

$$
\begin{gathered}
\partial_{t} r_{\varepsilon, \delta}+\operatorname{div}_{x}\left(r_{\varepsilon, \delta} \mathbf{U}_{\varepsilon, \delta}\right)=\left(\tilde{\varrho}_{\varepsilon}-1\right) \operatorname{div}_{x} \mathbf{V}_{\varepsilon, \delta}+\nabla_{x} \tilde{\varrho}_{\varepsilon} \cdot \mathbf{U}_{\varepsilon, \delta}+ \\
\varepsilon^{m} \partial_{t} q_{\varepsilon, \delta}+\varepsilon^{m} \operatorname{div}_{x}\left(\left(s_{\varepsilon, \delta}+q_{\varepsilon, \delta}\right)\left(\mathbf{v}_{\varepsilon, \delta}+\mathbf{V}_{\varepsilon, \delta}\right)\right) .
\end{gathered}
$$


and

$$
\begin{gathered}
\varrho_{\varepsilon} \nabla_{x} H^{\prime}\left(r_{\varepsilon, \delta}\right) \cdot\left(\mathbf{U}_{\varepsilon, \delta}-\mathbf{u}_{\varepsilon}\right)-\nabla_{x} H^{\prime}\left(\tilde{\varrho}_{\varepsilon}\right) \cdot\left(r_{\varepsilon, \delta} \mathbf{U}_{\varepsilon, \delta}-\varrho_{\varepsilon} \mathbf{u}_{\varepsilon}\right) \\
=\varrho_{\varepsilon} \nabla_{x}\left[H^{\prime}\left(r_{\varepsilon, \delta}\right)-H^{\prime \prime}\left(\tilde{\varrho}_{\varepsilon}\right)\left(r_{\varepsilon, \delta}-\tilde{\varrho}_{\varepsilon}\right)-H^{\prime}\left(\tilde{\varrho}_{\varepsilon}\right)\right] \cdot\left(\mathbf{U}_{\varepsilon, \delta}-\mathbf{u}_{\varepsilon}\right) \\
+\varrho_{\varepsilon} \nabla_{x} H^{\prime \prime}\left(\tilde{\varrho}_{\varepsilon}\right) \cdot\left(\mathbf{U}_{\varepsilon, \delta}-\mathbf{u}_{\varepsilon}\right)\left(r_{\varepsilon, \delta}-\tilde{\varrho}_{\varepsilon}\right)+\varrho_{\varepsilon} \varepsilon^{m} H^{\prime \prime}\left(\tilde{\varrho}_{\varepsilon}\right)\left(\nabla_{x} s_{\varepsilon, \delta}+\nabla_{x} q_{\varepsilon, \delta}\right) \cdot\left(\mathbf{U}_{\varepsilon, \delta}-\mathbf{u}_{\varepsilon}\right) \\
+\left(\varrho_{\varepsilon}-r_{\varepsilon, \delta}\right) \nabla_{x} H^{\prime}\left(\tilde{\varrho}_{\varepsilon}\right) \cdot \mathbf{U}_{\varepsilon, \delta} .
\end{gathered}
$$

Here we have used the fact that the term $\varrho_{\varepsilon} \nabla_{x} H^{\prime}\left(\tilde{\varrho}_{\varepsilon}\right) \cdot\left(\mathbf{U}_{\varepsilon, \delta}-\mathbf{u}_{\varepsilon}\right)$ in the expansion of $\varrho_{\varepsilon} \nabla_{x} H^{\prime}\left(r_{\varepsilon, \delta}\right)$. $\left(\mathbf{U}_{\varepsilon, \delta}-\mathbf{u}_{\varepsilon}\right)$ cancels with the same term in the expansion of $\nabla_{x} H^{\prime}\left(\varrho_{\varepsilon}\right) \cdot\left(r_{\varepsilon, \delta} \mathbf{U}_{\varepsilon, \delta}-\varrho_{\varepsilon} \mathbf{u}_{\varepsilon}\right)$.

Now we use formula (6.21) with the second and third terms at the right hand side expressed

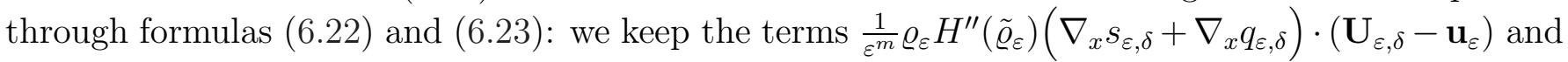
$\frac{1}{\varepsilon^{m}}\left(r_{\varepsilon, \delta}-\varrho_{\varepsilon}\right) \nabla_{x} H^{\prime}\left(r_{\varepsilon, \delta}\right)\left[\partial_{t} q_{\varepsilon, \delta}+\operatorname{div}_{x}\left(\left(s_{\varepsilon, \delta}+q_{\varepsilon, \delta}\right)\left(\mathbf{v}_{\varepsilon, \delta}+\mathbf{V}_{\varepsilon, \delta}\right)\right)\right]$ as they are, and estimate the decay of all remaining terms as $\varepsilon \rightarrow 0$.

We observe that, thanks to (6.9), (6.12),

$$
\sup _{t \in(0, T)}\left\|q_{\varepsilon, \delta}(t, \cdot)\right\|_{L^{2}\left(R^{2}\right)} \leq c, \sup _{t \in(0, T)}\left\|\Delta q_{\varepsilon, \delta}(t, \cdot)\right\|_{L^{2}\left(R^{2}\right)} \leq c \varepsilon^{m-1}, \sup _{t \in(0, T)}\left\|\partial_{t} q_{\varepsilon, \delta}(t, \cdot)\right\|_{L^{2}\left(R^{2}\right)} \leq c \varepsilon^{m-1} .
$$

Now, we use (2.4 2.5), (4.14.4), (6.5), (6.8), (6.13), (6.24) to deduce the following estimates:

$$
\begin{gathered}
\frac{1}{\varepsilon^{2 n}}\left|\int_{0}^{\tau} \int_{\Omega}\left(r_{\varepsilon, \delta}-\varrho_{\varepsilon}\right) \nabla_{x} G \cdot \mathbf{U}_{\varepsilon, \delta} \mathrm{d} x \mathrm{~d} t\right| \leq c \varepsilon^{m-2 n} \\
\frac{1}{\varepsilon^{2 m}} \int_{0}^{\tau} \int_{\Omega}\left[p\left(r_{\varepsilon, \delta}\right)-p^{\prime}\left(r_{\varepsilon, \delta}\right)\left(r_{\varepsilon, \delta}-\varrho_{\varepsilon}\right)-p\left(\varrho_{\varepsilon}\right)\right] \operatorname{div}_{x} \mathbf{U}_{\varepsilon, \delta} \mathrm{d} x \mathrm{~d} t \\
=\frac{1}{\varepsilon^{2 m}} \int_{0}^{\tau} \int_{\Omega}\left[p\left(r_{\varepsilon, \delta}\right)-p^{\prime}\left(r_{\varepsilon, \delta}\right)\left(r_{\varepsilon, \delta}-\varrho_{\varepsilon}\right)-p\left(\varrho_{\varepsilon}\right)\right]_{\operatorname{res}} \operatorname{div}_{x} \mathbf{V}_{\varepsilon, \delta} \mathrm{d} x \mathrm{~d} t \\
+\frac{1}{\varepsilon^{2 m}} \int_{0}^{\tau} \int_{\Omega}\left[p\left(r_{\varepsilon, \delta}\right)-p^{\prime}\left(r_{\varepsilon, \delta}\right)\left(r_{\varepsilon, \delta}-\varrho_{\varepsilon}\right)-p\left(\varrho_{\varepsilon}\right)\right]_{\operatorname{ess}} \operatorname{div}_{x} \mathbf{V}_{\varepsilon, \delta} \mathrm{d} x \mathrm{~d} t=h(\varepsilon, \delta), \\
\frac{1}{\varepsilon^{2 m}}\left|\int_{0}^{\tau} \int_{\Omega}\left(r_{\varepsilon, \delta}-\varrho_{\varepsilon}\right) H^{\prime \prime}\left(r_{\varepsilon, \delta}\right)\left(\left(\varrho_{\varepsilon}-1\right) \operatorname{div}_{x} \mathbf{V}_{\varepsilon, \delta}+\nabla_{x} \tilde{\varrho}_{\varepsilon} \cdot \mathbf{U}_{\varepsilon, \delta}\right) \mathrm{d} x \mathrm{~d} t\right| \leq c \varepsilon^{m-2 n}, \\
\frac{1}{\varepsilon^{2 m}} \int_{0}^{\tau} \int_{\Omega} \varrho_{\varepsilon} \nabla_{x}\left[H^{\prime}\left(r_{\varepsilon, \delta}\right)-H^{\prime \prime}\left(\varrho_{\varepsilon}\right)\left(r_{\varepsilon, \delta}-\tilde{\varrho}_{\varepsilon}\right)-H^{\prime}\left(\tilde{\varrho}_{\varepsilon}\right)\right] \cdot\left(\mathbf{U}_{\varepsilon, \delta}-\mathbf{u}_{\varepsilon}\right) \mathrm{d} x \mathrm{~d} t=h(\varepsilon, \delta),
\end{gathered}
$$


$\frac{1}{\varepsilon^{2 m}}\left(\left|\int_{\Omega} \varrho_{\varepsilon} \nabla_{x} H^{\prime \prime}\left(\tilde{\varrho}_{\varepsilon}\right) \cdot\left(\mathbf{U}_{\varepsilon, \delta}-\mathbf{u}_{\varepsilon}\right)\left(r_{\varepsilon, \delta}-\tilde{\varrho}_{\varepsilon}\right) \mathrm{d} x \mathrm{~d} t\right|+\left|\int_{0}^{\tau} \int_{\Omega}\left(\varrho_{\varepsilon}-r_{\varepsilon, \delta}\right) \nabla_{x} H^{\prime}\left(\tilde{\varrho}_{\varepsilon}\right) \cdot \mathbf{U}_{\varepsilon, \delta} \mathrm{d} x \mathrm{~d} t\right| \leq c \varepsilon^{m-2 n}\right.$.

Here and hereafter, $h(\varepsilon, \delta)$ denotes a generic function having the property

$$
h(\varepsilon, \delta) \rightarrow \tilde{h}(\delta) \text { as } \varepsilon \rightarrow 0, \tilde{h}(\delta) \rightarrow 0 \text { as } \delta \rightarrow 0 .
$$

Note that the dispersive decay estimates (6.8) play a crucial role in the analysis.

Taking into account (6.19), (6.20), using the identity (6.21) with the third and fourth terms expressed through (6.22 6.23), and employing the asymptotic behavior from formulas (6.25 6.29), we may rewrite the relative entropy inequality (2.12) in the form

$$
\begin{aligned}
\mathcal{E}_{\varepsilon}\left(\varrho_{\varepsilon}, \mathbf{u}_{\varepsilon} \mid r_{\varepsilon, \delta}, \mathbf{U}_{\varepsilon, \delta}\right)(\tau) & \leq \int_{0}^{\tau} \int_{\Omega} \varrho_{\varepsilon}\left(\partial_{t} \mathbf{U}_{\varepsilon, \delta}+\mathbf{u}_{\varepsilon} \cdot \nabla_{x} \mathbf{U}_{\varepsilon, \delta}\right) \cdot\left(\mathbf{U}_{\varepsilon, \delta}-\mathbf{u}_{\varepsilon}\right) \mathrm{d} x \mathrm{~d} t \\
& +\frac{1}{\varepsilon} \int_{0}^{\tau} \int_{\Omega} \varrho_{\varepsilon}\left(\mathbf{f} \times \mathbf{u}_{\varepsilon}\right) \cdot\left(\mathbf{U}_{\varepsilon, \delta}-\mathbf{u}_{\varepsilon}\right) \mathrm{d} x \mathrm{~d} t \\
+ & \frac{1}{\varepsilon^{m}} \int_{0}^{\tau} \int_{\Omega}\left(r_{\varepsilon, \delta}-\varrho_{\varepsilon}\right) H^{\prime \prime}\left(r_{\varepsilon, \delta}\right)\left[\partial_{t} q_{\varepsilon, \delta}+\operatorname{div}_{x}\left(\left(s_{\varepsilon, \delta}+q_{\varepsilon, \delta}\right) \mathbf{U}_{\varepsilon, \delta}\right)\right] \mathrm{d} x \mathrm{~d} t \\
+ & \frac{1}{\varepsilon^{m}} \int_{0}^{\tau} \int_{\Omega} \varrho_{\varepsilon} H^{\prime \prime}\left(\tilde{\varrho}_{\varepsilon}\right)\left(\nabla_{x} s_{\varepsilon, \delta}+\nabla_{x} q_{\varepsilon, \delta}\right) \cdot\left(\mathbf{U}_{\varepsilon, \delta}-\mathbf{u}_{\varepsilon}\right) \mathrm{d} x \mathrm{~d} t+h(\varepsilon, \delta) .
\end{aligned}
$$

\subsubsection{Coriolis force}

We may write

$$
\begin{gathered}
\varrho_{\varepsilon} H^{\prime \prime}\left(\tilde{\varrho}_{\varepsilon}\right)\left(\nabla_{x} s_{\varepsilon, \delta}+\nabla_{x} q_{\varepsilon, \delta}\right) \cdot\left(\mathbf{U}_{\varepsilon, \delta}-\mathbf{u}_{\varepsilon}\right) \\
=\varrho_{\varepsilon}\left(H^{\prime \prime}\left(\tilde{\varrho}_{\varepsilon}\right)-H^{\prime \prime}(1)\right)\left(\nabla_{x} s_{\varepsilon, \delta}+\nabla_{x} q_{\varepsilon, \delta}\right) \cdot\left(\mathbf{U}_{\varepsilon, \delta}-\mathbf{u}_{\varepsilon}\right)+\varrho_{\varepsilon} H^{\prime \prime}(1)\left(\nabla_{x} s_{\varepsilon, \delta}+\nabla_{x} q_{\varepsilon, \delta}\right) \cdot\left(\mathbf{U}_{\varepsilon, \delta}-\mathbf{u}_{\varepsilon}\right),
\end{gathered}
$$

where, by the same reasoning as in estimates (6.25 6.29),

$$
\frac{1}{\varepsilon^{2 m}}\left|\int_{0}^{\tau} \int_{\Omega} \varrho_{\varepsilon}\left(H^{\prime \prime}\left(\varrho_{\varepsilon}\right)-H^{\prime \prime}(1)\right)\left(\nabla_{x} s_{\varepsilon, \delta}+\nabla_{x} q_{\varepsilon, \delta}\right) \cdot\left(\mathbf{U}_{\varepsilon, \delta}-\mathbf{u}_{\varepsilon}\right) \mathrm{d} x \mathrm{~d} t\right| \leq c \varepsilon^{m-2 n}
$$

Recalling our convention $H^{\prime \prime}(1)=1$ we get for the second term in (6.32),

$$
\begin{gathered}
\frac{1}{\varepsilon^{m}} \varrho_{\varepsilon} H^{\prime \prime}(1)\left(\nabla_{x} s_{\varepsilon, \delta}+\nabla_{x} q_{\varepsilon, \delta}\right) \cdot\left(\mathbf{U}_{\varepsilon, \delta}-\mathbf{u}_{\varepsilon}\right)= \\
\varrho_{\varepsilon}\left(-\partial_{t} \mathbf{V}_{\varepsilon, \delta}-\frac{1}{\varepsilon} \mathbf{f} \times \mathbf{V}_{\varepsilon, \delta}-\frac{1}{\varepsilon} \mathbf{f} \times \mathbf{v}_{\varepsilon, \delta}\right) \cdot\left(\mathbf{U}_{\varepsilon, \delta}-\mathbf{u}_{\varepsilon}\right) \\
=-\partial_{t} \mathbf{V}_{\varepsilon, \delta}-\frac{1}{\varepsilon}\left(\mathbf{f} \times \mathbf{U}_{\varepsilon, \delta}\right) \cdot \mathbf{u}_{\varepsilon},
\end{gathered}
$$


where the last term cancels out with the Coriolis force term $\frac{1}{\varepsilon} \mathbf{f} \times \mathbf{u}_{\varepsilon} \cdot\left(\mathbf{U}_{\varepsilon, \delta}-\mathbf{u}_{\varepsilon}\right)=\frac{1}{\varepsilon}\left(\mathbf{f} \times \mathbf{u}_{\varepsilon}\right) \cdot \mathbf{U}_{\varepsilon, \delta}$.

Thanks to formula (6.32) the relative entropy inequality (6.31) reduces to

$$
\begin{aligned}
& \mathcal{E}_{\varepsilon}\left(\varrho_{\varepsilon}, \mathbf{u}_{\varepsilon} \mid r_{\varepsilon, \delta}, \mathbf{U}_{\varepsilon, \delta}\right)(\tau) \leq \int_{0}^{\tau} \int_{\Omega} \varrho_{\varepsilon}\left(\partial_{t} \mathbf{v}_{\varepsilon, \delta}+\mathbf{u}_{\varepsilon} \cdot \nabla_{x} \mathbf{U}_{\varepsilon, \delta}\right) \cdot\left(\mathbf{U}_{\varepsilon, \delta}-\mathbf{u}_{\varepsilon}\right) \mathrm{d} x \mathrm{~d} t \\
& +\frac{1}{\varepsilon^{m}} \int_{0}^{\tau} \int_{\Omega}\left(r_{\varepsilon, \delta}-\varrho_{\varepsilon}\right) H^{\prime \prime}\left(r_{\varepsilon, \delta}\right)\left[\partial_{t} q_{\varepsilon, \delta}+\operatorname{div}_{x}\left(\left(s_{\varepsilon, \delta}+q_{\varepsilon, \delta}\right) \mathbf{U}_{\varepsilon, \delta}\right)\right] \mathrm{d} x \mathrm{~d} t+h(\varepsilon, \delta) .
\end{aligned}
$$

\subsubsection{Estimating the remaining terms}

We observe that

$$
\begin{gathered}
\int_{0}^{\tau} \int_{\Omega} \varrho_{\varepsilon}\left(\partial_{t} \mathbf{v}_{\varepsilon, \delta}+\mathbf{u}_{\varepsilon} \cdot \nabla_{x} \mathbf{U}_{\varepsilon, \delta}\right) \cdot\left(\mathbf{U}_{\varepsilon, \delta}-\mathbf{u}_{\varepsilon}\right) \mathrm{d} x \mathrm{~d} t=\int_{0}^{\tau} \int_{\Omega} \varrho_{\varepsilon}\left(\mathbf{v}_{\varepsilon, \delta}+\mathbf{U}_{\varepsilon, \delta} \cdot \nabla_{x} \mathbf{U}_{\varepsilon, \delta}\right) \cdot\left(\mathbf{U}_{\varepsilon, \delta}-\mathbf{u}_{\varepsilon}\right) \mathrm{d} x \mathrm{~d} t \\
-\int_{0}^{\tau} \int_{\Omega} \varrho_{\varepsilon}\left(\mathbf{U}_{\varepsilon, \delta}-\mathbf{u}_{\varepsilon}\right) \cdot \nabla_{x} \mathbf{U}_{\varepsilon, \delta} \cdot\left(\mathbf{U}_{\varepsilon, \delta}-\mathbf{u}_{\varepsilon}\right) \mathrm{d} x \mathrm{~d} t,
\end{gathered}
$$

where the terms in the first expression at the right hand side containing the quantities $s_{\varepsilon, \delta}, \mathbf{V}_{\varepsilon, \delta}$ tend

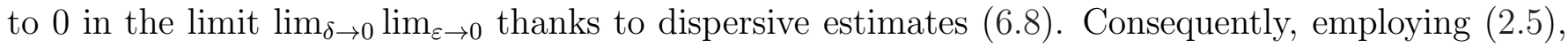
we obtain

$$
\begin{gathered}
\int_{0}^{\tau} \int_{\Omega} \varrho_{\varepsilon}\left(\partial_{t} \mathbf{v}_{\varepsilon, \delta}+\mathbf{u}_{\varepsilon} \cdot \nabla_{x} \mathbf{U}_{\varepsilon, \delta}\right) \cdot\left(\mathbf{U}_{\varepsilon, \delta}-\mathbf{u}_{\varepsilon}\right) \mathrm{d} x \mathrm{~d} t \\
\leq \int_{0}^{\tau} \int_{\Omega}\left(\partial_{t} \mathbf{v}_{\varepsilon, \delta}+\mathbf{v}_{\varepsilon, \delta} \cdot \nabla_{x} \mathbf{v}_{\varepsilon, \delta}\right) \cdot\left(\mathbf{v}_{\varepsilon, \delta}-\mathbf{u}_{\varepsilon}\right) \mathrm{d} x \mathrm{~d} t+c \int_{0}^{\tau} \mathcal{E}_{\varepsilon}\left(\varrho_{\varepsilon}, \mathbf{u}_{\varepsilon} \mid r_{\varepsilon, \delta}, \mathbf{U}_{\varepsilon, \delta}\right) \mathrm{d} t+h(\varepsilon, \delta) .
\end{gathered}
$$

Similarly, we deduce

$$
\begin{gathered}
\frac{1}{\varepsilon^{m}} \int_{0}^{\tau} \int_{\Omega}\left(r_{\varepsilon, \delta}-\varrho_{\varepsilon}\right) H^{\prime \prime}\left(r_{\varepsilon, \delta}\right)\left[\partial_{t} q_{\varepsilon, \delta}+\operatorname{div}_{x}\left(\left(s_{\varepsilon, \delta}+q_{\varepsilon, \delta}\right) \mathbf{U}_{\varepsilon, \delta}\right)\right] \mathrm{d} x \mathrm{~d} t \\
=\int_{0}^{\tau} \int_{\Omega} q_{\varepsilon, \delta} \partial_{t} q_{\varepsilon, \delta} \mathrm{d} x \mathrm{~d} t+h(\varepsilon, \delta)
\end{gathered}
$$

where we have used (4.14.3), (6.6), (6.24).

Now, we employ the energy equality (6.12) to observe that

$$
\int_{0}^{\tau} \int_{\Omega}\left(\mathbf{v}_{\varepsilon, \delta} \cdot \partial_{t} \mathbf{v}_{\varepsilon, \delta}+q_{\delta, \varepsilon} \cdot \partial_{t} q_{\varepsilon, \delta}\right) \mathrm{d} x \mathrm{~d} t=0
$$

Consequently,

$$
\begin{gathered}
\int_{0}^{\tau} \int_{\Omega}\left(\partial_{t} \mathbf{v}_{\varepsilon, \delta}+\mathbf{v}_{\varepsilon, \delta} \cdot \nabla_{x} \mathbf{v}_{\varepsilon, \delta}\right) \cdot\left(\mathbf{v}_{\varepsilon, \delta}-\mathbf{u}_{\varepsilon}\right) \mathrm{d} x \mathrm{~d} t \\
+\int_{0}^{\tau} \int_{\Omega} q_{\varepsilon, \delta} \partial_{t} q_{\varepsilon, \delta} \mathrm{d} x \mathrm{~d} t=-\int_{0}^{\tau} \int_{\Omega}\left(\partial_{t} \mathbf{v}_{\varepsilon, \delta}+\mathbf{v}_{\varepsilon, \delta} \cdot \nabla_{x} \mathbf{v}_{\varepsilon, \delta}\right) \cdot \mathbf{u}_{\varepsilon} \mathrm{d} x \mathrm{~d} t
\end{gathered}
$$


where we have used the identity $\int_{0}^{\tau} \int_{\Omega} \mathbf{v}_{\varepsilon, \delta} \cdot \nabla_{x} \mathbf{v}_{\varepsilon, \delta} \cdot \mathbf{v}_{\varepsilon, \delta} \mathrm{d} x \mathrm{~d} t=0$.

We remark that

$$
\begin{gathered}
\int_{0}^{\tau} \int_{\Omega}\left(\partial_{t} \mathbf{v}_{\varepsilon, \delta}+\mathbf{v}_{\varepsilon, \delta} \cdot \nabla_{x} \mathbf{v}_{\varepsilon, \delta}\right) \cdot \mathbf{u}_{\varepsilon} \mathrm{d} x \mathrm{~d} t \rightarrow \int_{0}^{\tau} \int_{\Omega}\left(\partial_{t} \mathbf{v}_{\delta}+\mathbf{v}_{\delta} \cdot \nabla_{x} \mathbf{v}_{\delta}\right) \cdot \mathbf{u} \mathrm{d} x \mathrm{~d} t \\
=\int_{0}^{\tau} \int_{\Omega} \nabla_{x} \Pi_{\delta} \cdot \mathbf{u} \mathrm{d} x \mathrm{~d} t=0,
\end{gathered}
$$

where we have used (4.74.8), (6.15) and equations (1.9) with $(\mathbf{v}, \Pi)$ replaced by $\left(\mathbf{v}_{\delta}, \Pi_{\delta}\right)$. Consequently, the entropy inequality (6.33) takes the form

$$
\mathcal{E}_{\varepsilon}\left(\varrho_{\varepsilon}, \mathbf{u}_{\varepsilon} \mid r_{\varepsilon, \delta}, \mathbf{U}_{\varepsilon, \delta}\right)(\tau) \leq h(\varepsilon, \delta),
$$

where the function $h$ satisfies (6.30). This finishes the proof of Theorem 3.1

\section{References}

[1] A. Babin, A. Mahalov, and B. Nicolaenko. Global regularity of 3D rotating Navier-Stokes equations for resonant domains. Indiana Univ. Math. J., 48:1133-1176, 1999.

[2] A. Babin, A. Mahalov, and B. Nicolaenko. 3D Navier-Stokes and Euler equations with initial data characterized by uniformly large vorticity. Indiana Univ. Math. J., 50 (Special Issue):1-35, 2001.

[3] D. Bresch, B. Desjardins, and D. Gerard-Varet. Rotating fluids in a cylinder. Disc. Cont. Dyn. Syst., 11:47-82, 2004.

[4] J.-Y. Chemin, B. Desjardins, I. Gallagher, and E. Grenier. Mathematical geophysics, volume 32 of Oxford Lecture Series in Mathematics and its Applications. The Clarendon Press Oxford University Press, Oxford, 2006.

[5] T. Clopeau, A. Mikelić, and R. Robert. On the vanishing viscosity limit for the 2D incompressible Navier-Stokes equations with the friction type boundary conditions. Nonlinearity, 11(6):16251636, 1998.

[6] R. Danchin. Low Mach number limit for viscous compressible flows. M2AN Math. Model Numer. Anal., 39:459-475, 2005.

[7] D.R. Durran. Is the Coriolis force really responsible for the inertial oscillation ? Bull. Amer. Meteorological Soc., 74:2179-2184, 1993. 
[8] D. B. Ebin. The motion of slightly compressible fluids viewed as a motion with strong constraining force. Ann. Math., 105:141-200, 1977.

[9] E. Feireisl, I. Gallagher, and A. Novotný. A singular limit for compressible rotating fluids. SIAM J. Math. Anal., 44:192-205, 2012.

[10] E. Feireisl, Bum Ja Jin, and A. Novotný. Relative entropies, suitable weak solutions, and weakstrong uniqueness for the compressible Navier-Stokes system. J. Math. Fluid Mech., 14:712-730, 2012 .

[11] E. Feireisl and A. Novotný. Scale interactions in compressible rotating fluids. Anal. Mat. Pura Appl., 2013. Submitted.

[12] E. Feireisl, A. Novotný, and H. Petzeltová. On the existence of globally defined weak solutions to the Navier-Stokes equations of compressible isentropic fluids. J. Math. Fluid Mech., 3:358-392, 2001.

[13] E. Feireisl, Antonín Novotný, and Y. Sun. Suitable weak solutions to the Navier-Stokes equations of compressible viscous fluids. Indiana Univ. Math. J., 60(2):611-631, 2011.

[14] I. Gallagher. Résultats récents sur la limite incompressible. Astérisque, (299):Exp. No. 926, vii, 29-57, 2005. Séminaire Bourbaki. Vol. 2003/2004.

[15] P. Germain. Weak-strong uniqueness for the isentropic compressible Navier-Stokes system. $J$. Math. Fluid Mech., 13(1):137-146, 2011.

[16] Z. Guo, L. Peng, and B. Wang. Decay estimates for a class of wave equations. J. Funct. Anal., 254(6):1642-1660, 2008.

[17] T. Kato. Remarks on the zero viscosity limit for nonstationary Navier-Stokes flows with boundary. In Seminar on PDE's, S.S. Chern (ed.), Springer, New York, 1984.

[18] T. Kato and C.Y. Lai. Nonlinear evolution equations and the Euler flow. J. Funct. Anal., 56:15-28, 1984.

[19] S. Klainerman and A. Majda. Singular limits of quasilinear hyperbolic systems with large parameters and the incompressible limit of compressible fluids. Comm. Pure Appl. Math., 34:481-524, 1981.

[20] R. Klein. Scale-dependent models for atmospheric flows. In Annual review of fluid mechanics. Vol. 42, Annu. Rev. Fluid. Mech., pages 249-274. Annual Reviews, Palo Alto, CA, 2010. 
[21] P.-L. Lions. Mathematical topics in fluid dynamics, Vol.2, Compressible models. Oxford Science Publication, Oxford, 1998.

[22] P.-L. Lions and N. Masmoudi. Incompressible limit for a viscous compressible fluid. J. Math. Pures Appl., 77:585-627, 1998.

[23] N. Masmoudi. The Euler limit of the Navier-Stokes equations, and rotating fluids with boundary. Arch. Rational Mech. Anal., 142:375-394, 1998.

[24] N. Masmoudi. Incompressible, inviscid limit of the compressible Navier-Stokes system. Ann. Inst. Henri Poincaré, Anal. non linéaire, 18:199-224, 2001.

[25] N. Masmoudi. Examples of singular limits in hydrodynamics. In Handbook of Differential Equations, III, C. Dafermos, E. Feireisl Eds., Elsevier, Amsterdam, 2006.

[26] M. Oliver. Classical solutions for a generalized Euler equation in two dimensions. J. Math. Anal. Appl., 215:471-484, 1997.

[27] M. Sammartino and R. Caflisch. Zero viscosity limit for analytic solutions of the Navier-Stokes equation on a half-space I, Existence for Euler and Prandtl equations. Commun. Math. Phys., 192:433-461, 1998.

[28] M. Sammartino and R. Caflisch. Zero viscosity limit for analytic solutions of the Navier-Stokes equation on a half-space II, Construction of the Navier-Stokes solution. Commun. Math. Phys., 192:463-491, 1998.

[29] S. Schochet. The mathematical theory of low Mach number flows. M2ANMath. Model Numer. anal., 39:441-458, 2005.

[30] E.M. Stein. Singular integrals and differential properties of functions. Princeton University Press, Princeton, 1970.

[31] H.S.G. Swann. The convergence with vanishing viscosity of nonstationary Navier-Stokes flow to ideal flow in $R^{3}$. Trans. Amer. Math. Soc., 157:373-397, 1971.

[32] R. Temam and X. Wang. On the behavior of the solutions of the Navier-Stokes equations at vanishing viscosity. Annali Scuola Normale Pisa, 25:807-828, 1997.

[33] R. Temam and X. Wang. Boundary layers associated with incompressible NavierStokes equations: The noncharacteristic boundary case. J. Differential Equations, 179:647-686, 2002.

[34] G.K. Vallis. Atmospheric and oceanic fluid dynamics. Cambridge University Press, Cambridge, 2006. 